\title{
Rainfall Characteristics of Recurving Tropical Cyclones over the Western North Pacific
}

\author{
Difei Deng a AND Elizabeth A. Ritchie \\ School of Physical, Environmental and Mathematical Sciences, University of New South Wales, \\ Canberra, Australian Capital Territory, Australia
}

(Manuscript received 18 June 2017, in final form 2 October 2017)

\begin{abstract}
A dataset of 88 recurving western North Pacific tropical cyclones from 2004 to 2015 is investigated for rainfall characteristics during their period of recurvature. The TCs are categorized into two groups based on different large-scale patterns from empirical orthogonal function analysis. Group 1 is characterized by an intense midlatitude baroclinic zone and close distance between the zone and TC, while Group 2 is characterized by a weaker midlatitude baroclinic zone and more remote distance between the zone and TC at the time of recurvature. The results show the large-scale environment has substantial impact on TC rainfall patterns. In Group 1, as the TC approaches and is embedded into the baroclinic zone, a relatively strong interaction between the TC and midlatitudes occurs, which is reflected by a rapid increase of environmental vertical wind shear and TC translation speed, the alignment of the shear vector and motion vector, and a sharp contrast of temperature and moisture. Higher rainfall and wider coverage of rainfall tends to be produced along the track after recurvature, and the rainfall pattern turns from a right-of-track (ROT) to a left-of-track (LOT) preference. Conversely, in Group 2, a relatively weak interaction between the TC and midlatitude circulation occurs, which is reflected by weaker vertical wind shear and slower TC motion, a separation of the shear vector and motion vector, and a weak gradient of temperature and moisture. The corresponding rainfall swath for Group 2 exhibits a narrower rainfall swath after recurvature. The rain pattern changes from a LOT to ROT preference.
\end{abstract}

\section{Introduction}

Predicting rainfall associated with tropical cyclones (TCs) is a major operational challenge (Lonfat et al. 2004), and the spatial distribution of rainfall in TCs is of particular interest for the quantitative precipitation forecast (Chen et al. 2006). Increasing evidence has shown that TC rainfall, convection, and winds can vary dramatically from nearly symmetric to highly asymmetric under external environmental influences. Asymmetries in the TC rainfall have been studied both observationally and numerically for many years. Prior studies have found that asymmetries in TC circulations are mainly related to the gradient of planetary vorticity (Peng et al. 1999), the TC motion, the friction-induced asymmetric boundary layer convergence (Shapiro 1983), the environmental vertical wind shear (Jones 1995; 2000; Frank and Ritchie 1999,

\footnotetext{
${ }^{a}$ Former affiliation: Institute of Atmospheric Physics, Chinese Academy of Sciences, Beijing, China.
}

Corresponding author: Difei Deng, ddfttkl@gmail.com
2001), and possibly the asymmetric moisture distribution surrounding a storm (Chan et al. 2004).

The wavenumber- 1 asymmetry in circulation, induced by interaction between the symmetric circulation and the planetary vorticity gradient, not only causes a general northwestward motion of the TC but also increases the inward radial flux in the southeast quadrant in a zero-mean flow environment on a beta plane (Madala and Piacsek 1975). Local shear of nearly $5 \mathrm{~m} \mathrm{~s}^{-1}$ can be produced by beta gyres alone as the accompanying beta gyres also produce flow across the vortex from the southeast, which weakens with height. The upward motion induced by this "beta shear" would be expected to be downshear in a quiescent background environment (Bender 1997; Ritchie and Frank 2007). With the addition of mean flow, the maximum inward radial flux shifts to the southwest quadrant in the presence of a westerly mean flow and, conversely, to the east side of the TC in an easterly mean flow (Peng et al. 1999).

In addition to the beta effect, TC motion can also modulate the TC rainfall pattern. Although the amount and exact location of rainfall varies from storm to storm, the 
observed rainfall generally has a maximum ahead of the TC center, which is caused by surface friction-induced boundary inflow and low-level convergence. Shapiro (1983) used a simple boundary layer model to analyze the effects of storm movement on the boundary frictional convergence and found that for slow-moving storms the convergence was concentrated in the front-right quadrant, whereas for fastmoving storms it was located more directly in front. Corbosiero and Molinari (2003) provided a detailed review of the effect of storm motion on TC structure and rainfall in the eyewall $(r \leq 30 \mathrm{~km})$, inner rainband $(30<r \leq 100 \mathrm{~km})$, and outer rainband $(r>100 \mathrm{~km})$. Generally, the asymmetry in precipitation occurred preferentially in the front quadrants of the core and inner band regions, with a slight preference for the right of motion. Rainfall at larger radii was more to the right of the track, with the maximum in the front-right quadrant. Some more recent studies highlighted the effect of geographic location and TC intensity on the motion-relative TC rainfall distribution. A long-term statistical study of TC rainfall showed that the wavenumber-1 rainfall maximum tended to be located in the front to frontright quadrant in the western North Pacific, and the frontright quadrant in the North Atlantic Ocean (Chen et al. 2006). Lonfat et al. (2004) indicated that the maximum rainfall shifted from front left for weak storms (with surface maximum wind less than $33 \mathrm{~m} \mathrm{~s}^{-1}$ ) to front right for strong storms (with surface maximum wind larger than $49 \mathrm{~m} \mathrm{~s}^{-1}$ ) in the outer rainband regions.

Moderate changes in the environmental vertical wind shear (VWS) can also alter the storm in ways that could potentially affect the structure and intensity. In a dry ideal model, Jones (1995) showed that as the VWS began to tilt the vortex, a compensating secondary vertical circulation developed to be in balance with associated temperature anomalies, and this produced upward motion downshear and downward motion upshear. Frank and Ritchie (2001) found that the tilt of the potential vorticity (PV) column produced low-level convergence on the down-tilt side of the vortex when VWS was introduced in their idealized simulations, triggering a wavenumber-1 asymmetry in vertical motion, cloud water, and rainfall with a preference for upward motion and rainfall in the downshear-left quadrant. Similar results were also found in observational studies of Hurricanes Chantal and Erin (2001) (Halverson et al. 2006; Heymsfield et al. 2006). Rogers et al. (2003) simulated Hurricane Bonnie (1993) and found that across-track shear produced a rain swath that was evenly distributed across the track whereas along-track shear produced preferential left-of-track rain. Corbosiero and Molinari (2002) used lightening data and ECWMF reanalysis data to statistically analyze maxima in lightning, convective activity, and rainfall, and found that the strongest convection occurred downshear with a slight preference for downshear left in the inner core and a strong preference for downshear right in the outer rainbands.

Other environmental conditions, including dry air intrusion or completing extratropical transition (ET), may also influence the structure of a TC. For example, Klein et al. (2000) and Chan et al. (2004) demonstrated that the intrusion of dry continental air into a TC's circulation reduced rainfall and caused the rain shield to assume an asymmetrical shape. Ritchie and Elsberry (2001) depicted the main rainfall characteristic changes during the transformation stage of the extratropical transition of tropical cyclones in the Northern Hemisphere. The core convection and precipitation first weakened as the TC moved over the decreased sea surface temperatures and began to interact with the midlatitude baroclinic zone. Gradual erosion of clouds and deep convection in the west through south sectors of the TC occurred because of the intrusion of dry, cold midlatitude air. Deep subsidence on the west side of the TC due to the convergence between upperlevel midlatitude flow and the TC circulation also contributed to warming and cloud erosion as the TC approached the midlatitudes. Finally, the southern semicircle was almost completely clear of clouds and rainfall was constrained to the north of the TC. Finally, Atallah et al. (2007) showed that the precipitation in landfalling TCs on the east coast of the United States could be categorized as left of track (LOT) or right of track (ROT) depending on the strength of the midlatitude trough to the northeast of the TC.

These previous studies highlight the importance of environmental fields on TC structure and rainfall asymmetry. The western North Pacific is the most active TC basin globally with approximately 24 TCs occurring per year on average. Almost half of the TCs recurve back to the north and northeast (Jones et al. 2003; Kitabatake 2008; Kofron et al. 2010a,b). During recurvature, the TC moves from the deep tropical easterly regime to the strong uppertropospheric westerlies of the midlatitudes. During this transition significant environmental changes take place, including a reversal of the mean steering flow, an increase in the vertical wind shear, and amplification of meridional temperature and moisture gradients. However, relatively few quantitative studies have investigated how the changes in the environmental fields affect the asymmetric rainfall characteristics of the recurving TCs. This study focuses on the variation of the spatial extent and rainfall distribution of recurving TCs over the western North Pacific and investigates the effects of the environmental fields on the asymmetry of TC rainfall during recurvature.

The structure of the rest of the paper is as follows. The data and methodology used in the study are described in section 2. Characteristic rainfall patterns of recurving 
TCs in the western North Pacific are described in section 3. Section 4 presents an analysis of the large-scale patterns associated with the rainfall patterns and section 5 discusses how the large-scale environment affects the rainfall patterns. A discussion and conclusions are provided in section 5 .

\section{Data and methodology}

Recurving TCs in the western North Pacific are identified using the Japan Meteorological Agency (JMA) best-track dataset (http://www.jma.go.jp/jma/jma-eng/ jma-center/rsmc-hp-pub-eg/besttrack.html). All TCs whose tracks change from northwestward to north and northeastward are identified during the period 2004-15, resulting in a total dataset of 88 TCs. According to the JMA best-track reports, 81 of the 88 recurving TCs finally developed into an extratropical cyclone.

Global 6-hourly, $1^{\circ}$ final operational global analysis (FNL) gridded data from the Global Forecast System (GFS) (NCEP 2000) are used to examine the large-scale circulation patterns associated with the recurving TCs. For each recurving TC, the environmental fields are extracted from the FNL data, centered on the TC at the times from $24 \mathrm{~h}$ prior to the time of recurvature to $24 \mathrm{~h}$ after the time of recurvature, every $6 \mathrm{~h}$. The centered fields are $50^{\circ}$ latitude by $50^{\circ}$ longitude.

Tropical Rainfall Measuring Mission (TRMM) precipitation gridded product 3B42 (Kummerow et al. 2001; Huffman et al. 2001, 2007) at 3-hourly and $0.25^{\circ}$ resolution are obtained for the period 2004-15. These data are used to characterize the rainfall swath associated with each recurving TC. For each of the selected TCs, the 3-hourly rain rate within a 500-km radius of the JMA best-track TC center is extracted to ensure that only rain associated with the TC is used in the analysis and these values are then accumulated along the TC track to form the total rain swath.

Empirical orthogonal function (EOF) analysis (e.g., Bretherton et al. 1992) is used to objectively categorize the recurving TC cases based on the common large-scale patterns. EOF analysis has been successfully used to identify large-scale patterns associated with TC motion in the western North Pacific (e.g., Shaffer and Elsberry 1982; Harr et al. 2000), recurvature and extratropical transition (e.g., Sinclair 2004; Wood and Ritchie 2014), TC rapid weakening (Wood and Ritchie 2015), and TC size change (Stovern and Ritchie 2016). The EOF analysis is performed on the TC-centered $50^{\circ}$ latitude by $50^{\circ}$ longitude $500-\mathrm{hPa}$ geopotential fields at the time of recurvature. The first three EOF patterns are statistically significant and explain approximately $51 \%, 18 \%$, and $8 \%$ of the variance, respectively. Here, we focus on the analysis of the first EOF mode (EOF1) and PC1
(Fig. 1). The other modes will be analyzed in a future study.

Composite grids are created at three separate times, $24 \mathrm{~h}$ before recurvature $(T-24 \mathrm{~h})$, recurvature time $(T+0 \mathrm{~h})$, and $24 \mathrm{~h}$ after recurvature $(T+24 \mathrm{~h})$, for the large-scale circulation patterns using the TC-centered FNL grids. Composite rainfall grids are also created every $3 \mathrm{~h}$ from $24 \mathrm{~h}$ prior to recurvature to $24 \mathrm{~h}$ after recurvature using the TRMM 3B42 product.

\section{TC rainfall patterns associated with the two groups}

\section{a. The grouping of the TCs based on EOF1}

The first EOF explains just over $50 \%$ of the variance in the dataset while the second EOF explains $17 \%$ and the third EOF explains $8 \%$. These first three EOFs are all significant using the North test (North et al. 1982) (Fig. 1d). Here we investigate groupings based on the first EOF as this EOF appears to well represent the changes in the rainfall structure we observe. EOF1+ and EOF1- both present a midlatitude trough located to the northwest of the TC and a subtropical high pressure ridge to the east of the TC from the 500-hPa geopotential height (Figs. 1a,b). The gradient of geopotential height $5^{\circ}$ north of the TC center in EOF1+ is much stronger than that in EOF1-, suggesting that the intensity of the baroclinic zone in EOF1+ is stronger than that in EOF1 - at the time of recurvature. The baroclinic zone also extends closer to the TC in EOF1+ than in EOF1-. The normalized first principal component value (PC1) of the recurving TCs (Fig. 1c) can be used to divide the TCs into two groups. Group 1 contains 40 TCs with PC1 $>0$ and Group 2 contains 48 TCs with PC1 $<0$.

The tracks for the two groups are shown in Fig. 2 and Table 1 highlights some differences in the structural characteristics as the two groups of TCs become extratropical. The focus of this study is on the period around recurvature and not on ET and we note that most of the TCs in the study take more than $24 \mathrm{~h}$ after recurvature to complete ET. There are no significant differences in the TC intensity between the two groups (Table 1). However, Group 1 TCs do take less time to become asymmetric, develop a lower-level cold core, and fully transition into ex-TCs compared to Group 2 TCs, suggesting that the difference in the strength of the midlatitude circulation and distance away from the TC can result in different structural evolution of the TCs.

\section{b. The TC rainfall patterns in the two groups}

Using the 3-hourly TRMM precipitation dataset, the composite rainfall swaths for the TCs in the two groups 

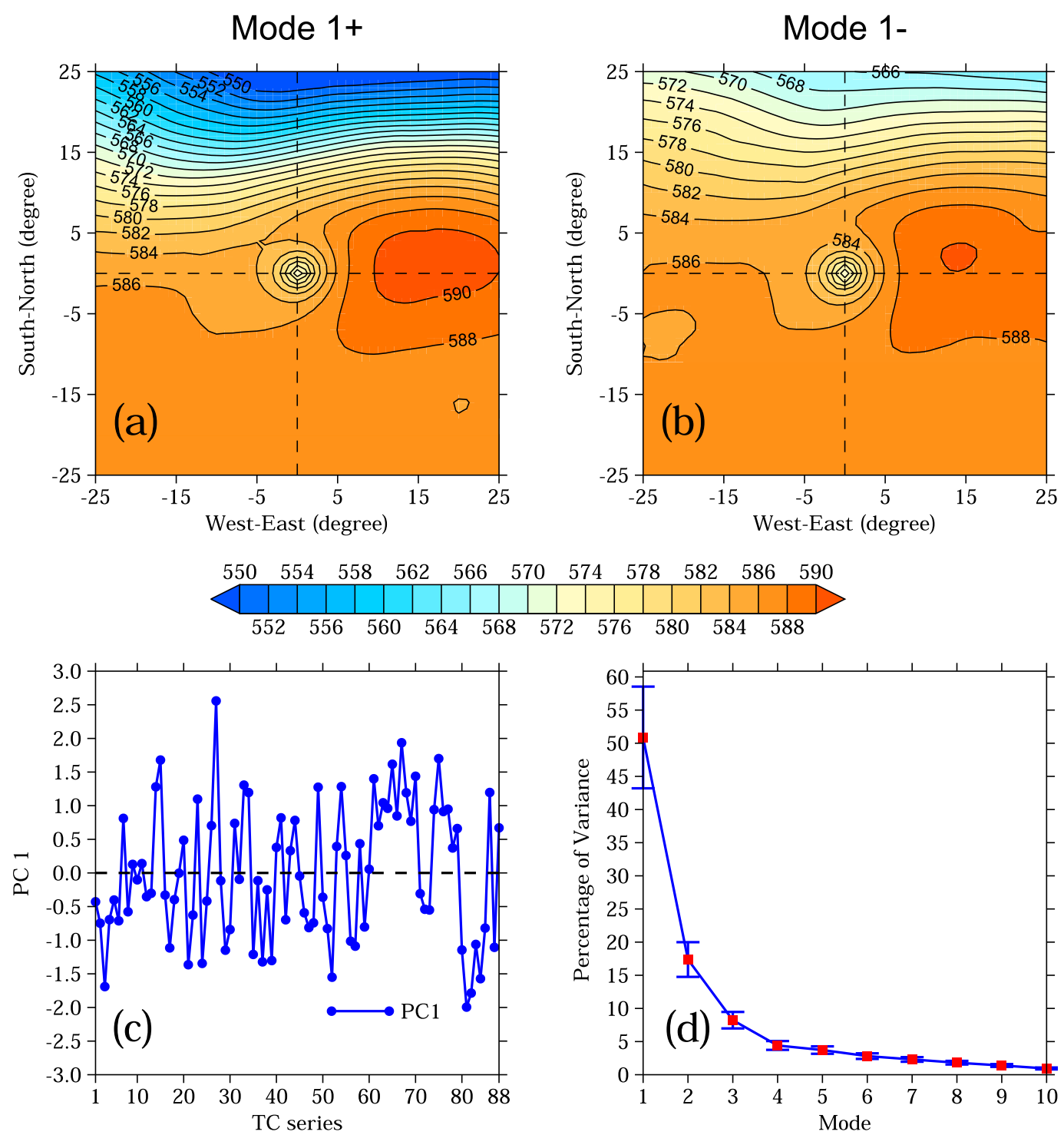

FIG. 1. TC-centered 500-hPa geopotential heights at the time of recurvature for (a) positive and (b) negative EOF 1 patterns, (c) the time series of the normalized PC value for the significant EOF1 mode, and (d) the explained variance and North test significance for the first $10 \mathrm{EOF}$ modes.

are shown in Fig. 3 from $T-24 \mathrm{~h}$ to $T+24 \mathrm{~h}$. There is more rainfall and wider spatial coverage of rainfall along the track after recurvature than before recurvature in Group 1 (Fig. 3a). Conversely, the rainfall swaths for Group 2 show less rainfall and a narrower rainfall extent after recurvature than before (Fig. 3b). Furthermore, the time series of average rainfall difference across the TC tracks highlights the evolution from ROT to LOT for Group 1 and from LOT to ROT for Group 2 TCs (Fig. 3).

The individual evolution of the composite rain rate for the two groups shows some additional information (Fig. 4). Group 1 rainfall (Figs. 4a-c) evolves from an initially axisymmetric (Fig. 4a) to elongated shape, with more rainfall concentrated ahead of the TC after recurvature. The rainfall moves to the LOT after recurvature (Fig. 4c), consistent with the time series in Fig. 3. In contrast, the Group 2 TC rainfall also evolves from initially axisymmetric to asymmetric at $T+24 \mathrm{~h}$, but with more rainfall to the ROT at the recurvature time (Fig. 4e) and $T+24 \mathrm{~h}$ (Fig. 4f). Moreover, the rainfall is diffused over a much larger area in Group 2 (Fig. 4f) than that in Group 1 (Fig. 4c) at $T+24 \mathrm{~h}$ with rain in the front-right and rear-right quadrants. The VWS and transition direction are denoted by a red arrow and a black arrow in Fig. 3, respectively. The specific values are in Table 2.

To quantify the evolution of the rainfall, time series of average rainfall within $500 \mathrm{~km}$ of the TC center are 
Group 1

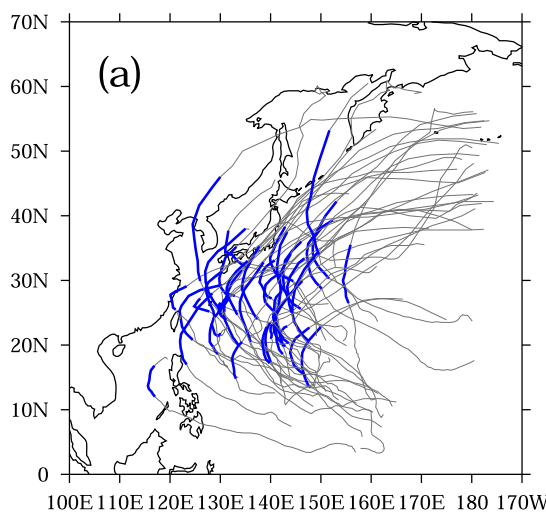

Group 2

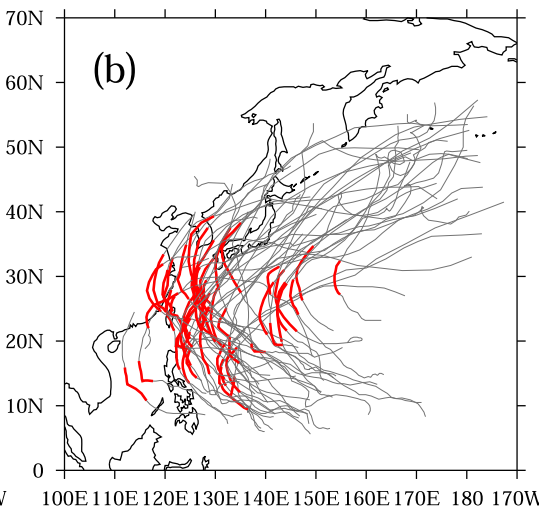

$T-24 h \sim T+24 h$

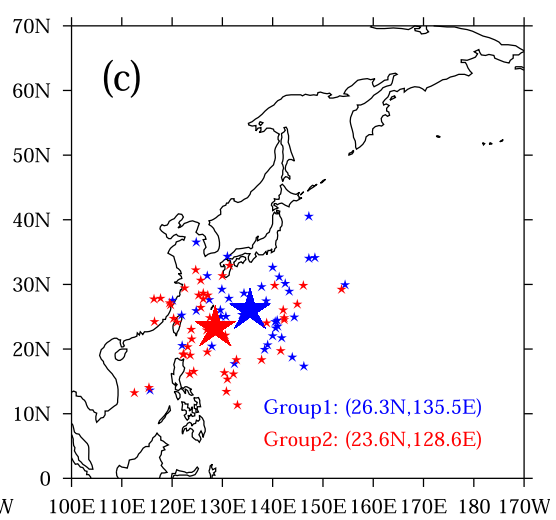

FIG. 2. The tracks with the 48-h period of the study highlighted for (a) Group 1 and (b) Group 2 TCs, and (c) the recurving points for Group 1 TCs (blue) and Group 2 TCs (red). The average recurvature points are denoted by the large blue star (Group 1) and red star (Group 2); the average latitude and longitude are indicated in the bottom-right corner in (c).

shown in Fig. 5. For the Group 1 TC, rainfall is initially distributed evenly among the four quadrants with a slight preference for the ROT quadrants (red solid line in Fig. 5a). Near the recurvature time the rainfall rapidly increases in the front-left quadrant so that by the end of the recurvature period the preference is clearly for LOT rainfall (blue solid line in Fig. 5a), maximized in the front-left quadrant. The rain switches from ROTdominant to LOT-dominant from about $T+3 \mathrm{~h}$. The Group 2 rainfall evolves quite differently with an initial preference for the rear quadrants, particularly the leftrear quadrant (LOT; blue solid line in Fig. 5b), which then rapidly decreases toward the recurvature time. At the same time the rainfall increases in the front-right quadrant and then after recurvature it rapidly increases in the front-left quadrant so that by the end of the recurvature period, rainfall is maximized in the two front quadrants with respect to TC motion with considerable rainfall also in the rear-right quadrant such that the maximum rainfall is ROT (red solid line in Fig. 5b). The solid lines show the switch from LOT-dominant to ROT-dominant rainfall from $12 \mathrm{~h}$ prior to recurvature $(T-12 \mathrm{~h})$ for the Group $2 \mathrm{TC}$.

\section{Large-scale patterns and TC features associated with groups 1 and 2}

In general, as the TC recurves, it approaches the midlatitudes where there is a midlatitude trough located to the northwest of the TC and a subtropical ridge to the east of the TC (Fig. 6). The baroclinic zone is stronger for the Group 1 TCs compared to Group 2, and the separation distance between the TC and baroclinic zone is less. For Group 1, the upper-level midlatitude westerly jet has core wind speeds of greater than $45 \mathrm{~m} \mathrm{~s}^{-1}$ and is located approximately $10^{\circ}$ north of the TC (shaded in Fig. 6a). During the recurvature period, the distance between the TC and jet decreases so that by the end of the recurvature period the jet and TC are separated by about $4^{\circ}$ and the jet core has increased to over $60 \mathrm{~m} \mathrm{~s}^{-1}$ (Figs. 6b,c). Upper-level divergence, which was located over the TC at $T-24 \mathrm{~h}$, extends into the rear-right entrance region of the jet and increases in strength (blue contours in Figs. 6a-c). The increase in jet strength and decrease in distance between the jet and TC results in a rapid increase in vertical wind shear from westerly at $5.5 \mathrm{~m} \mathrm{~s}^{-1}$ at the beginning of recurvature to westsouthwesterly at $7.3 \mathrm{~m} \mathrm{~s}^{-1}$ to southwesterly at $12 \mathrm{~m} \mathrm{~s}^{-1}$ by the end of the recurvature period (Table 2). By the end of the recurvature period the Group $1 \mathrm{TC}$ is fully embedded in the baroclinic zone.

In comparison, for Group 2 the jet is weaker $\left(35 \mathrm{~m} \mathrm{~s}^{-1}\right)$, and is located approximately $15^{\circ}$ north of the TC at the beginning of the recurvature period. The distance between the TC and jet decreases to $8^{\circ}$ by the end of the

TABLE 1. The time it takes from the recurvature point for the TC to reach asymmetric, lower-level (LL) cold core, and ex-TC status for Group 1 and Group 2 TCs. The values in boldface denote that the differences between the two groups are statistically significantly at the $95 \%$ confidence level using the Student's $t$ test. The values in parentheses are the standard deviation.

\begin{tabular}{llc}
\hline \hline Group & Variables & Time (h) \\
\hline Group 1 & Asymmetry & $\mathbf{2 7 . 0}(23.4)$ \\
& LL cold core & $\mathbf{5 2 . 9}(29.4)$ \\
Group 2 & Ex-TC & $\mathbf{5 4 . 8}(27.1)$ \\
& Asymmetry & $\mathbf{4 4 . 3}(22.7)$ \\
& LL cold core & $\mathbf{6 9 . 2}(40.2)$ \\
& Ex-TC & $\mathbf{6 5 . 8}(21.0)$ \\
\hline
\end{tabular}




\section{Group 1}

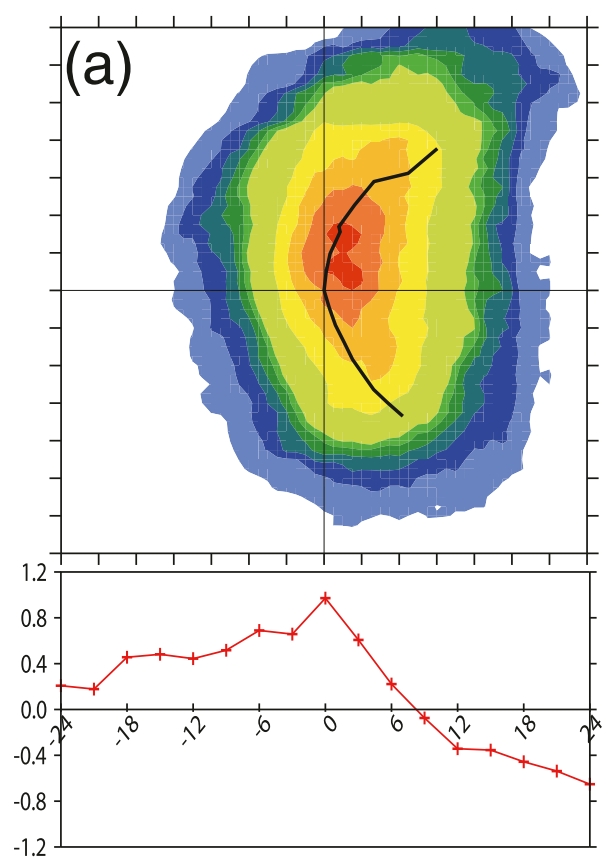

Group 2

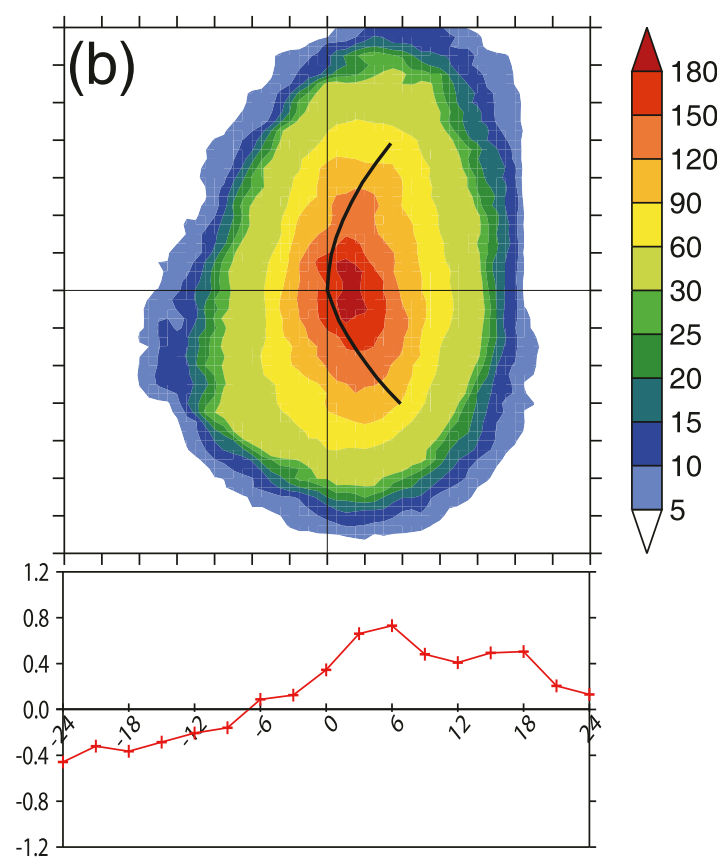

FIG. 3. Composite accumulated rainfall swaths $(\mathrm{mm})$ from TRMM during the $24 \mathrm{~h}$ before and $24 \mathrm{~h}$ after recurvature for (a) Group 1 and (b) Group 2 within a 500-km radius. The composite track is denoted by the black solid line. The domain is a $14^{\circ}$ latitude by $14^{\circ}$ longitude box. The bottom panels show the time series of the TC-motionrelative rain difference for each group, which is calculated by subtracting the average rain rate of the TC in a semicircle of radius $500 \mathrm{~km}$ to the right of motion with the semicircle to the left of motion.

recurvature period, and the jet speed increases to $40 \mathrm{~m} \mathrm{~s}^{-1}$ (Figs. 6d-f). The upper-level divergence is mostly associated with the tropical cyclone and only a weak connection to the jet exists (Fig. 6f). Moreover, the VWS rotates cyclonically from northeasterly to northwesterly and finally to westerly as the TC approaches the jet. The VWS is weak in Group 2, varying between $6.0 \mathrm{~m} \mathrm{~s}^{-1}$ at $T-24 \mathrm{~h}$ to $9.2 \mathrm{~m} \mathrm{~s}^{-1}$ at $T+24 \mathrm{~h}$ (Table 2).

The low-level temperature and moisture fields are shown in Fig. 7, highlighting the thermal trough (ridge) located to the west (east) of the TC. The temperature gradient (red contours), which is indicative of the midlatitude baroclinic zone, is stronger in Group 1 than in Group 2. As the Group 1 TC approaches and becomes embedded in the baroclinic zone, the temperature gradient increases around the TC circulation (Figs. $7 \mathrm{a}-\mathrm{c}$ ). The relative humidity (shaded) indicates that midlatitude dry air has already advected around the west of the TC at $24 \mathrm{~h}$ prior to recurvature, further intrudes around the south of the TC at the recurvature time, and has completely cut off the tropical moisture source to the south by the end of the recurvature period. Weak southwesterly flow is located to the south of the TC, indicating the weak interaction between the TC and tropical regions. The translation speed for Group 1 TCs increases from moderate before recurvature $\left(5.6 \mathrm{~m} \mathrm{~s}^{-1}\right.$ at $T-24 \mathrm{~h})$ to fast after recurvature $\left(9.9 \mathrm{~m} \mathrm{~s}^{-1}\right.$ at $\left.T+24 \mathrm{~h}\right)$ during transition (Table 2). By comparison, for Group 2 the midlatitude baroclinic zone is much weaker and located father north, there is less advection of midlatitude dry air around the west side of the TC, and the southwesterly flow to the south of the TC (indicated by the red dots) is stronger (Figs. 7d-f). Warm, tropical moisture appears to be transported into the TC circulation from the south and may contribute to the maintenance of rainfall in the southern semicircle of the Group 2 TC (Fig. 4f). Although the advection of the drier air from the north around the west side of the Group 2 TC is weaker than that in Group 1, this is likely still partially the cause of the decline in rainfall to the west of the Group 2 TC. Because of the weak interaction between the TC and midlatitude zone, the transition speed for Group 2 TCs is slower compared to Group 1, only $4.7 \mathrm{~m} \mathrm{~s}^{-1}$ at $T-24$ and $6.6 \mathrm{~m} \mathrm{~s}^{-1}$ at $T+24 \mathrm{~h}$ (Table 2).

The differences in the large-scale circulation between the Group 1 and Group 2 TCs are reflected not only in the magnitude of the VWS and motion vectors (Table 2) but also in their separation angle (Fig. 8). At $T-24 \mathrm{~h}$, the separation angles span the entire range from $-180^{\circ}$ 

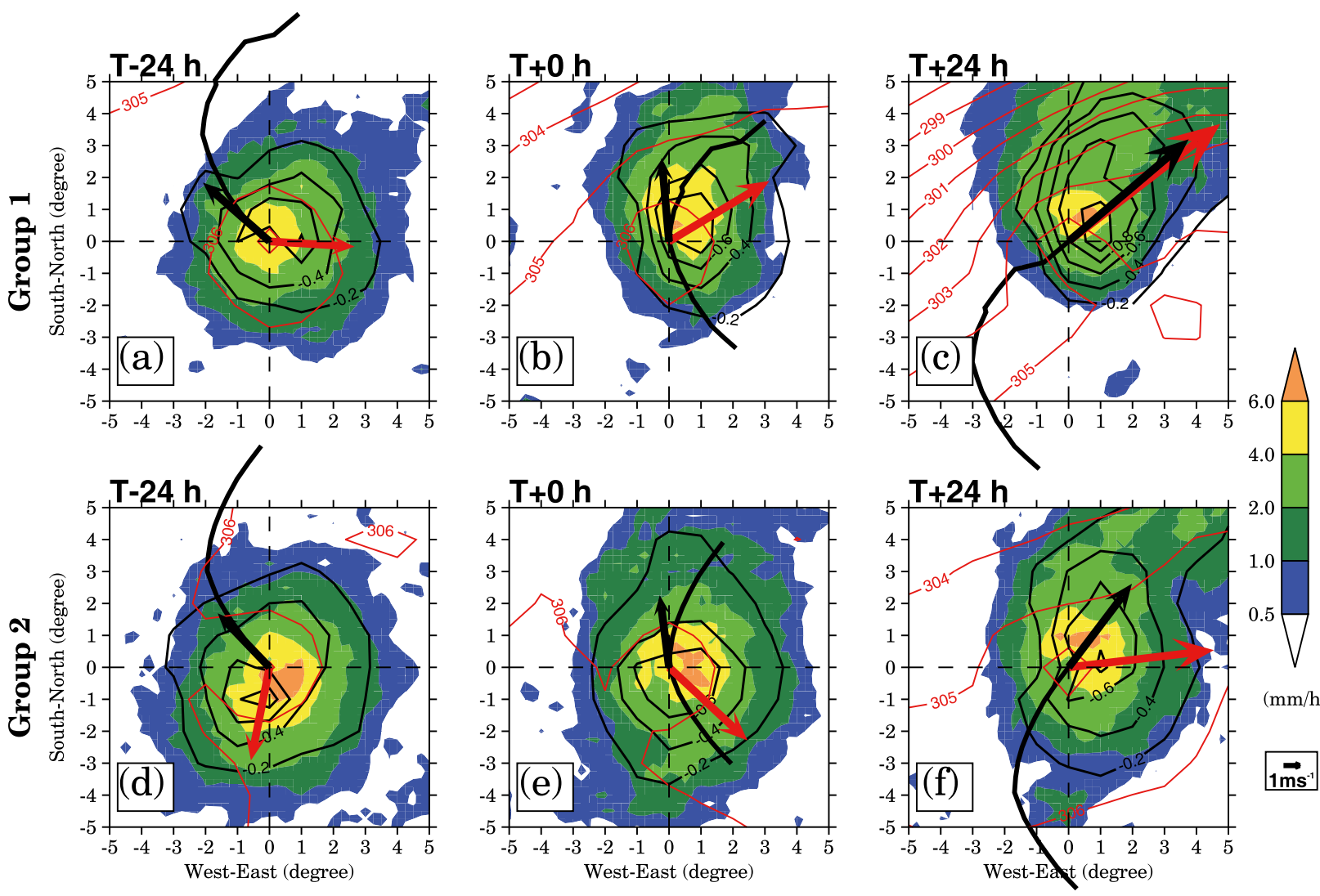

FIG. 4. The TRMM rain rate (shaded; $\mathrm{mm} \mathrm{h}^{-1}$ ), vertical velocity (black contour; $\mathrm{Pa} \mathrm{s}{ }^{-1}$ ), and 850-hPa potential temperature (red contours; contour interval is $1 \mathrm{~K}$ ) at (a),(d) $T-24 \mathrm{~h}$, (b),(e) $T+0 \mathrm{~h}$, and (c),(f) $T+24 \mathrm{~h}$ for (top) Group 1 and (bottom) Group 2 . The composite track is denoted by a black solid line, and the VWS vector and motion vector are denoted by the red arrow and black arrow, respectively.

to $+180^{\circ}$, with a slight preference between $0^{\circ}$ and $180^{\circ}$ for the Group 1 TCs and from $-180^{\circ}$ to $0^{\circ}$ for the Group 2 TCs because the lower-level southwesterly winds in Group 2 are stronger than the those in Group 1 before recurvature. At $T+0 \mathrm{~h}$, the majority of the angles in Group 1 concentrate between $0^{\circ}$ and $100^{\circ}$ as the Group 1 TCs quickly approach the midlatitude, whereas the angles for Group 2 are more highly distributed. At $T+24 \mathrm{~h}$, the shear and motion vectors are well aligned in Group 1, peaking at $0^{\circ}$ with a mean of $2.5^{\circ}$. For comparison, most of the angles in Group 2 become positive values (left-of-shear motion), with two peaks at $15^{\circ}$ and $90^{\circ}$. The separation angles between the two vectors are significantly different and robust (at a $95 \%$ confidence level) throughout the recurving period.

\section{Environmental influences on the vertical motion field and rainfall pattern}

The horizontal distribution of upward velocity (black contours in Fig. 4) is generally very similar to the horizontal distribution of rainfall, although the maximum rainfall center is located slightly to the left of maximum ascent center as the vortex flow moves cyclonically. As the rainfall distribution is directly

TABLE 2. The averaged VWS magnitude and TC motion speed for Group 1 and Group 2 TCs. Boldfaced values are statistically significantly at the $95 \%$ confidence level using the Student's $t$ test. The values in parentheses are the standard deviation.

\begin{tabular}{|c|c|c|c|c|c|c|}
\hline \multirow{2}{*}{$\begin{array}{c}\text { Variables } \\
\text { Groups }\end{array}$} & \multicolumn{2}{|c|}{$T-24 \mathrm{~h}$} & \multicolumn{2}{|c|}{$T+0 \mathrm{~h}$} & \multicolumn{2}{|c|}{$T+24 \mathrm{~h}$} \\
\hline & Group 1 & Group 2 & Group 1 & Group 2 & Group 1 & Group 2 \\
\hline VWS magnitude $\left(\mathrm{m} \mathrm{s}^{-1}\right)$ & $5.5(3.0)$ & $6.0(3.5)$ & $7.3(4.5)$ & $6.7(3.5)$ & $\mathbf{1 2 . 0}(7.8)$ & $9.2(5.0)$ \\
\hline Motion speed $\left(\mathrm{m} \mathrm{s}^{-1}\right)$ & $5.6(2.2)$ & $4.7(1.9)$ & $5.2(2.5)$ & $4.6(1.9)$ & $9.9(5.6)$ & $6.6(2.8)$ \\
\hline
\end{tabular}




\section{Group 1}

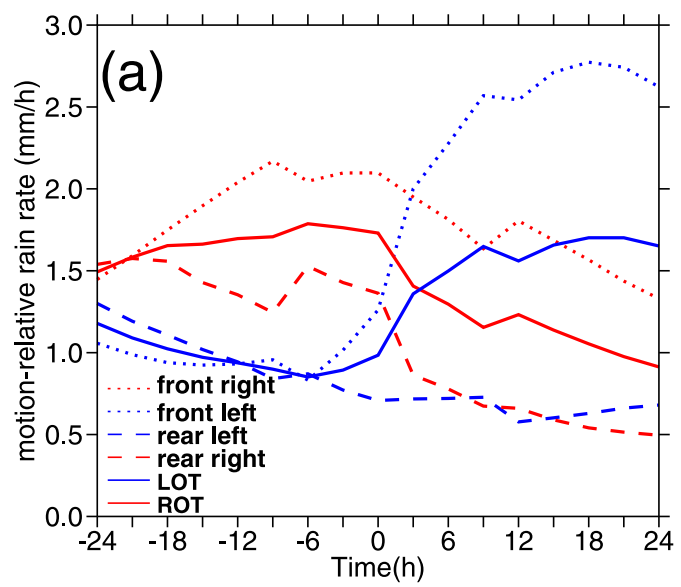

Group 2

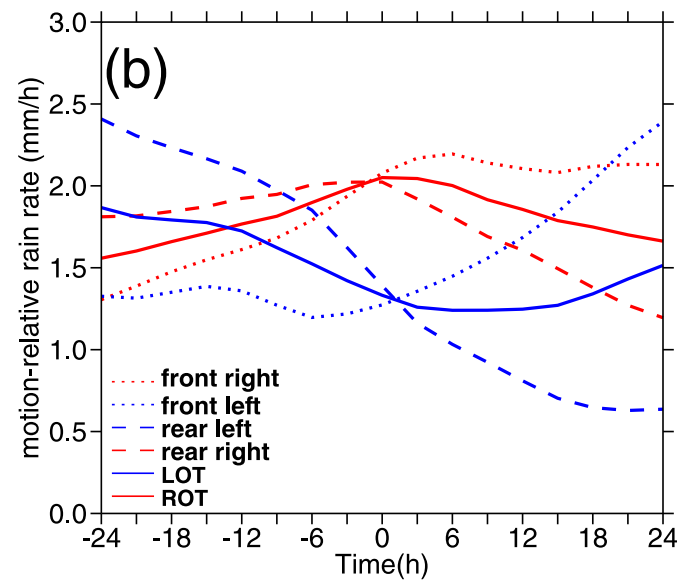

FIG. 5. The time series of area-averaged rain rate by quadrant relative to the TC motion within $500 \mathrm{~km}$ radius for (a) Group 1 and (b) Group 2. Left-of-track (LOT) rainfall is the sum of the rainfall in the front-left and rear-left quadrants. Right-of-track (ROT) rainfall is the sum of the rainfall in the front-right and rear-right quadrants.

related to the vertical motion field and moisture distribution, this section starts with a diagnosis of vertical motion around the TC circulation, and is followed by the diagnosis of atmospheric moisture.
There are limitations to this analysis owing to the relatively coarse resolution of the FNL gridded data and the upward motion in the inner core cannot be separated from the outer core in this analysis.
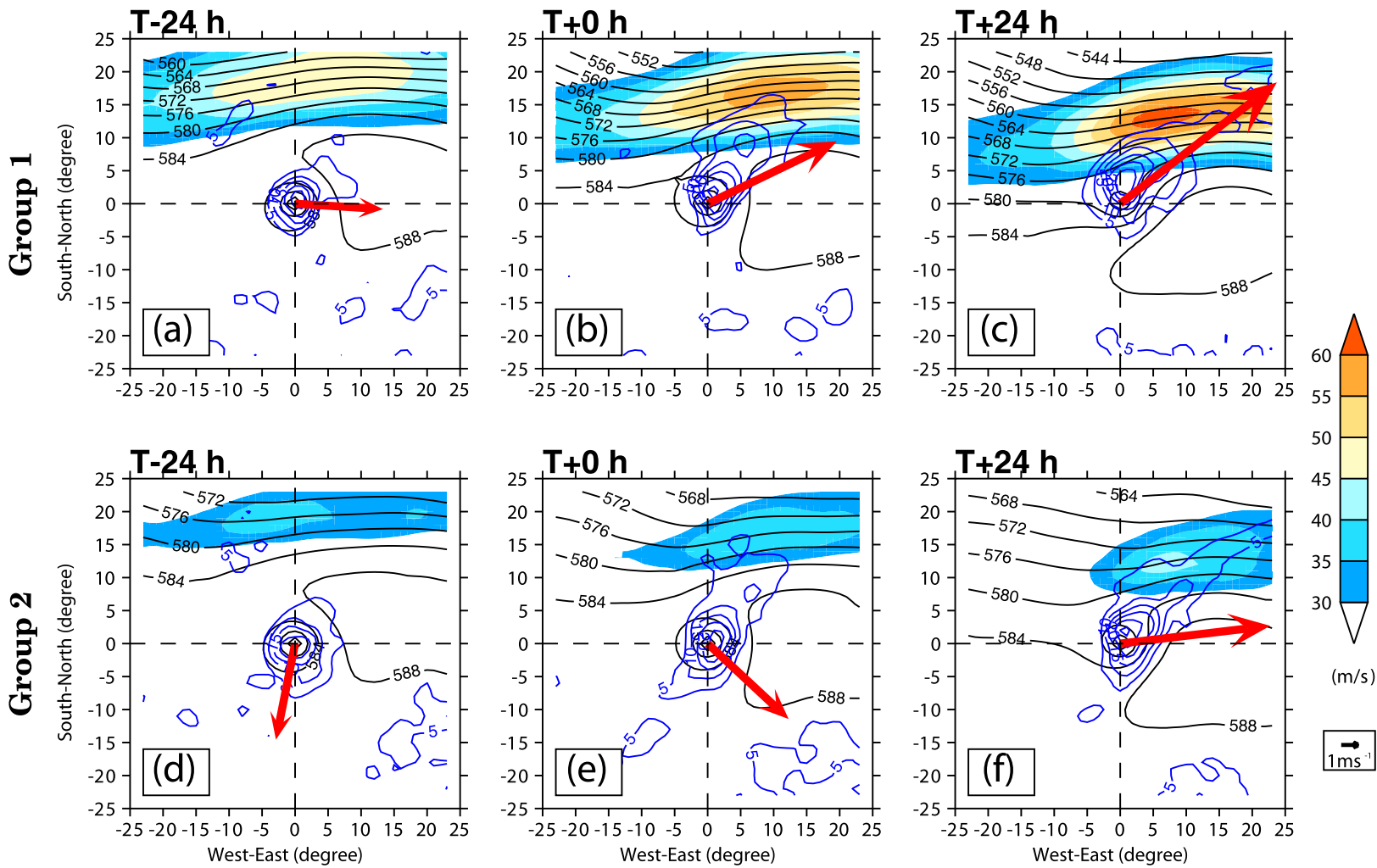

FIG. 6. TC-centered 500-hPa geopotential height (black contours; dam), 200-hPa wind field (shaded; wind speed larger than $30 \mathrm{~m} \mathrm{~s}^{-1}$ ) for (top) Group 1 and (bottom) Group 2 TCs at (a),(d) $T-24 \mathrm{~h}$, (b),(e) $T+0 \mathrm{~h}$, and (c),(f) $T+24 \mathrm{~h}$. The blue contours are the 200-hPa divergence $\left(10^{-6} \mathrm{~s}^{-1}\right)$. The vertical wind shear is denoted by the red arrow. 

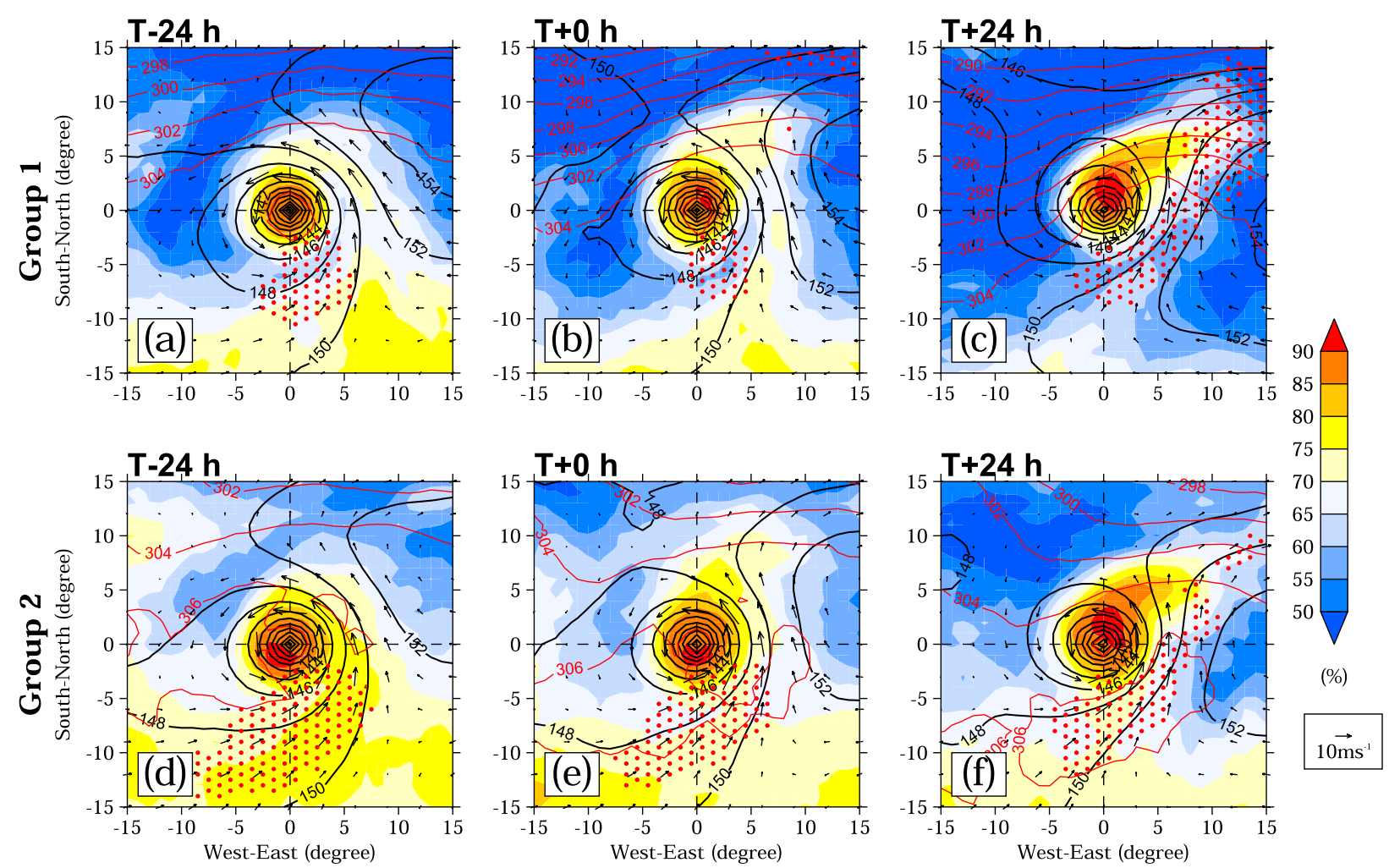

FIG. 7. TC-centered 850-hPa geopotential height (black contour; dam), wind field (vector), potential temperature (red contour; K), and 700-hPa relative humidity (shaded, \%) for groups (top) 1 and (bottom) 2 at (a),(d) $T-24 \mathrm{~h}$, (b),(e) $T+0 \mathrm{~h}$, and (c),(f) $T+24 \mathrm{~h}$. The dotted areas represent the 850 -hPa southwesterly wind larger than $6 \mathrm{~m} \mathrm{~s}^{-1}$.

Therefore, the focus of the study is to analyze the overall vertical motion and rainfall distribution within the TC circulation during the period of recurvature.

\section{a. Large-scale vertical wind shear}

Environmental vertical wind shear has been shown to influence the vertical motion pattern in the TC (e.g., Jones 1995; Frank and Ritchie 1999, 2001; Corbosiero and Molinari 2003) such that the preferential quadrant of convection and upward motion is located in the downshear- to downshear-left quadrants of the TC in the shear environment. From Table 2, the averaged environmental VWS of the Group 1 TC changes from westerly at $5.5 \mathrm{~m} \mathrm{~s}^{-1}$ to southwesterly at $12 \mathrm{~m} \mathrm{~s}^{-1}$ by the end of recurvature, while the VWS of the Group 2 TC changes less, from north-northeasterly at $6 \mathrm{~m} \mathrm{~s}^{-1}$ to westerly at $9.2 \mathrm{~m} \mathrm{~s}^{-1}$. The variations in the VWS affect the structure of the TC potential vorticity column and as a result have an effect on the vertical motion field (Jones 1995). At the beginning of recurvature (Fig. 9a), the VWS is weak in the Group 1 TCs and the PV column is well aligned in the vertical. By the recurvature time, the VWS has increased to $7.3 \mathrm{~m} \mathrm{~s}^{-1}$, and the PV column begins to tilt to the northeast (Fig. 9b) and by the end of recurvature the PV tilt is quite pronounced (Fig. 9c). Consistent with the increasing VWS and tilted PV anomaly, the profiles of VWS-relative vertical motion evolves from being relatively axisymmetric to asymmetric (Figs. 10a-c). Initially, the ascent is relatively evenly distributed among the four quadrants (Fig. 10a). After recurving, the ascent intensifies in the downshear quadrants and becomes negligible in the upshear quadrants (Figs. 10b,c) similar to the VWS-induced convection in Jones (1995) and Frank and Ritchie (1999, 2001).

In contrast, the VWS for the Group 2 TC is initially just strong enough to cause a slight tilt to the south and a slightly enhanced vertical motion field downshear (Fig. 9d). At the recurvature time, the PV column recovers to almost vertical, with a slight tilt to the southeast (Fig. 9e). It is not until the end of the recurvature period (Fig. 9f) that the Group 2 PV column tilts directly toward the east under the increased westerly VWS. The variation of the profiles of vertical motion by quadrant is less dramatic than that in Group 1 (Figs. 10d-f), but a similar pattern is observed after the environmental VWS exceeds $7 \mathrm{~m} \mathrm{~s}^{-1}$ (Fig. 10f) such that upward motion 

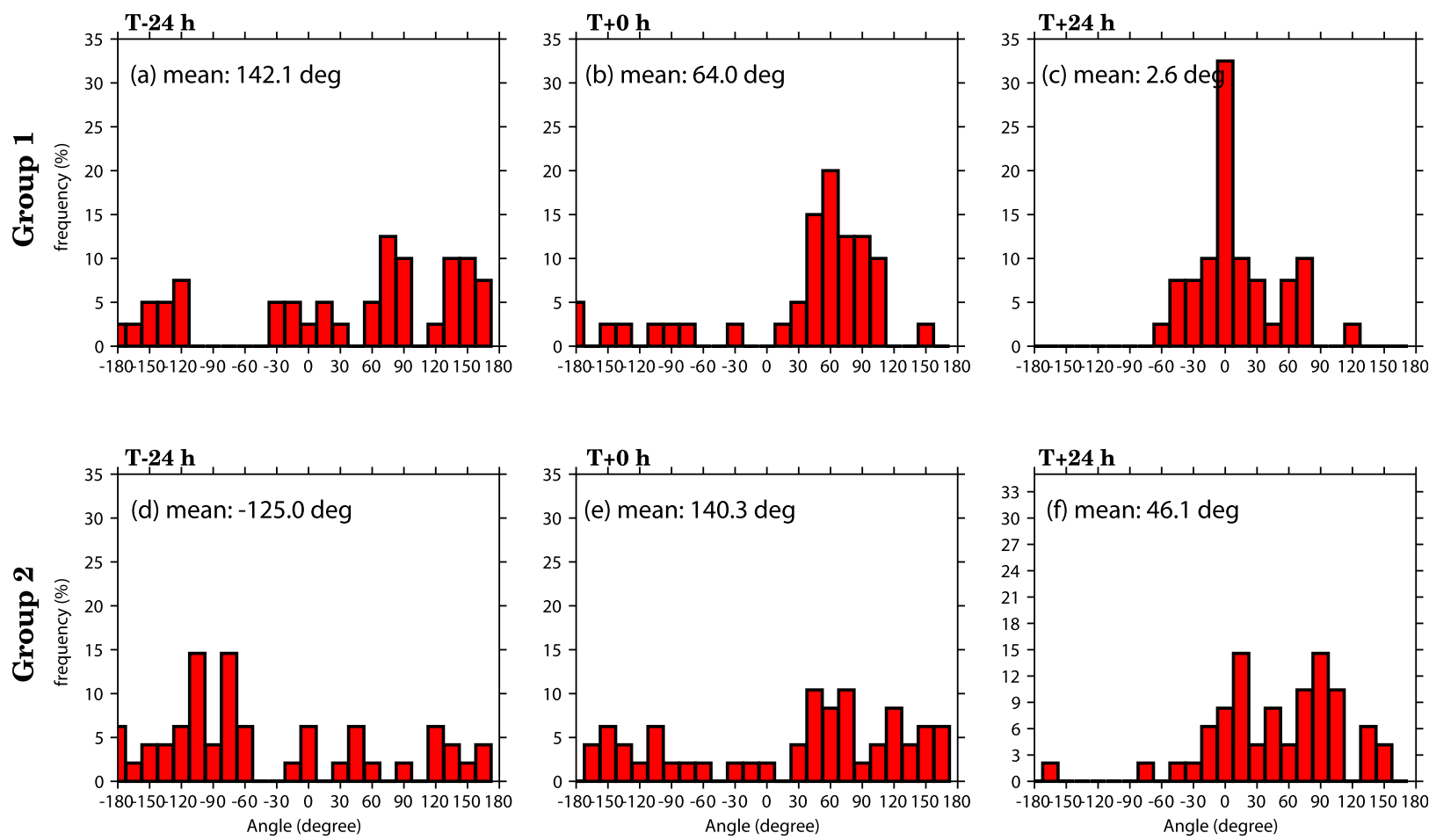

FIG. 8. Frequency distribution of the angle separation between the VWS and motion vectors (left-of-shear motion vector: from $0^{\circ}$ to $180^{\circ}$; right-of shear motion: from $-180^{\circ}$ to $0^{\circ}$ ) for Group 1 and 2 TCs. The mean value is indicated in the top-left corner of each panel. The differences in the angles between the two groups are significant at a 95\% confidence level using the Student's $t$ test.

is maximized in the downshear quadrants. Weak ascent can still be seen in the upshear quadrants.

\section{b. TC motion}

When TCs recurve, the reversal in the mean steering flow results in the TC moving from northwestward to northeastward. For a relatively slow-moving TC $\left(\leq 5 \mathrm{~m} \mathrm{~s}^{-1}\right)$, the frictional boundary layer convergence is located in a broad region in the front-right quadrant of the TC, while for a fast-moving storm $\left(\geq 10 \mathrm{~m} \mathrm{~s}^{-1}\right)$ convergence becomes concentrated directly ahead of the TC (Shapiro 1983). The maximum convection is typically observed in the front-right quadrant with respect to storm motion for both inner core and outer rainband (Corbosiero and Molinari 2003). Because of the relatively shallow vertical extent of the boundary friction effect, the vertical velocities triggered by the storm motion become much weaker above $700 \mathrm{hPa}$ unless reinforced by moist processes (Frank and Ritchie 1999).

The detailed structure of the boundary layer convergence cannot be resolved because of the coarse resolution of the reanalysis data although the main features can be seen in Fig. 11. In general, the distributions of convergence in the boundary layer in the two groups are consistent with the results of Shapiro (1983). The boundary convergence in Group 1 is a little stronger than
Group 2 at $T-24 \mathrm{~h}$, most likely because the TC translation speed in Group 1 is a little faster than Group 2.

The convergence in Group 1 changes from axisymmetric in a broad region initially to asymmetric during recurvature (Fig. 11a). At the recurvature time, the boundary layer convergence is to the right of the motion and then becomes concentrated ahead of the fastmoving $\mathrm{TC}$ at the end of the recurvature period (Figs. 11b,c). For Group 1, the motion-relative ascent by quadrant is initially evenly distributed in the four quadrants and corresponds to the axisymmetric horizontal boundary layer convergence (Fig. 12a). As the VWS is weaker than the TC motion speed at this time, the distribution of vertical motion resembles the ideal simulation of boundary friction effect (Fig. 3 in Frank and Ritchie 1999). As the TC recurves, the shear and motion vector become more aligned and increase in magnitude and it becomes difficult to completely separate the effects of the VWS and the frictional convergence due to the TC motion. Overall, the ascent intensifies in the front quadrants with a slight emphasis on the front-left quadrant by the end of recurvature (Fig. 12c), consistent with both the motion and shear vectors. Because of the alignment of the motion and shear vectors, these vertical motion patterns are reinforced. 

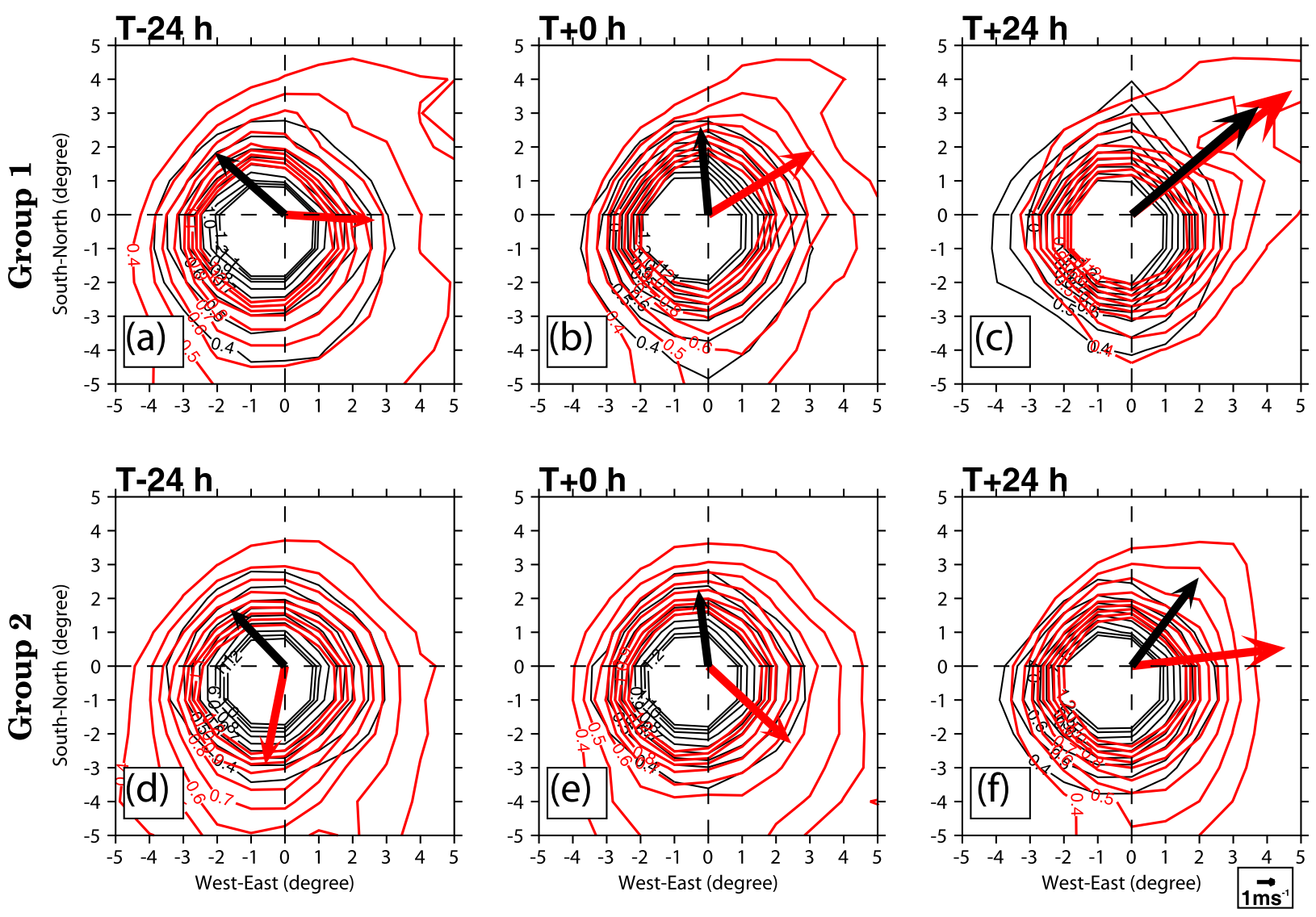

FIG. 9. 400-hPa (red contours) and 850-hPa PV (black contours) for (top) Group 1 and (bottom) Group 2 TCs at (a),(d) $T-24 \mathrm{~h}$, (b),(e) $T+0 \mathrm{~h}$, and (c),(f) $T+24 \mathrm{~h}$. The motion vector is denoted by the black arrow and the VWS vector is denoted by the red arrow.

For Group 2, the boundary layer convergence is weaker and less concentrated compared with Group 1 but the development of the spatial patterns is similar (Figs. 11e,f). The ascent is also initially evenly distributed in the four quadrants (Fig. 12d). As the TC recurves the motion-relative boundary layer convergence and upward motion moves to the front of the TC as reflected by the higher vertical motions in the front quadrants in Fig. 12f. However, the motion and shear vector remain misaligned throughout the period of recurvature and this is reflected in the additional ascent in the rear-right quadrant for all levels at $T+24 \mathrm{~h}$ relative to the motion vector, which is most likely contributed to by the VWS.

\section{c. Thermodynamic effect}

In addition to the dynamic lifting conditions, the environmental temperature and moisture fields also play important roles in modulating the rainfall pattern and intensity (e.g., Ritchie and Elsberry 2001, 2007; Jiang et al. 2008a,b). The Group 1 TCs are characterized by a small core of high specific humidity and lower amounts of moist air to the south and east of the TC, whereas the Group 2 TCs have a larger core of high specific humidity and higher amounts of moist air to the south and east of the TC (not shown). The pattern of moisture advection (black contours in Fig. 13) is similar for both groups of TCs. However, there is a larger area of negative moisture advection for the Group 1 TCs, particularly during and by the end of the recurvature period, which moves inward to the center of the TC, cutting off the tropical moisture supply from the south and eroding the TC core (Figs. 13a-c). The process is very similar to the dry slot in the transformation stage of ET (Klein et al. 2000). In Group 2, the moist air advection is much weaker and although it moves in toward the TC core with time, the TC remains well connected with the moisture source at lower latitudes (Figs. 13d-f).

The thermal advection fields are shown in the shading in Fig. 13. As the Group 1 TC approaches and interacts with the midlatitude baroclinic zone, a strong cold and warm advection dipole develops and intensifies, which resembles the cold and warm frontogenesis associated with the extratropical transition of TCs (Figs. 13a-c). The poleward flow associated with the TC circulation advects warm moist air to the northeast of the TC, contributing a sharpening of the temperature gradient 

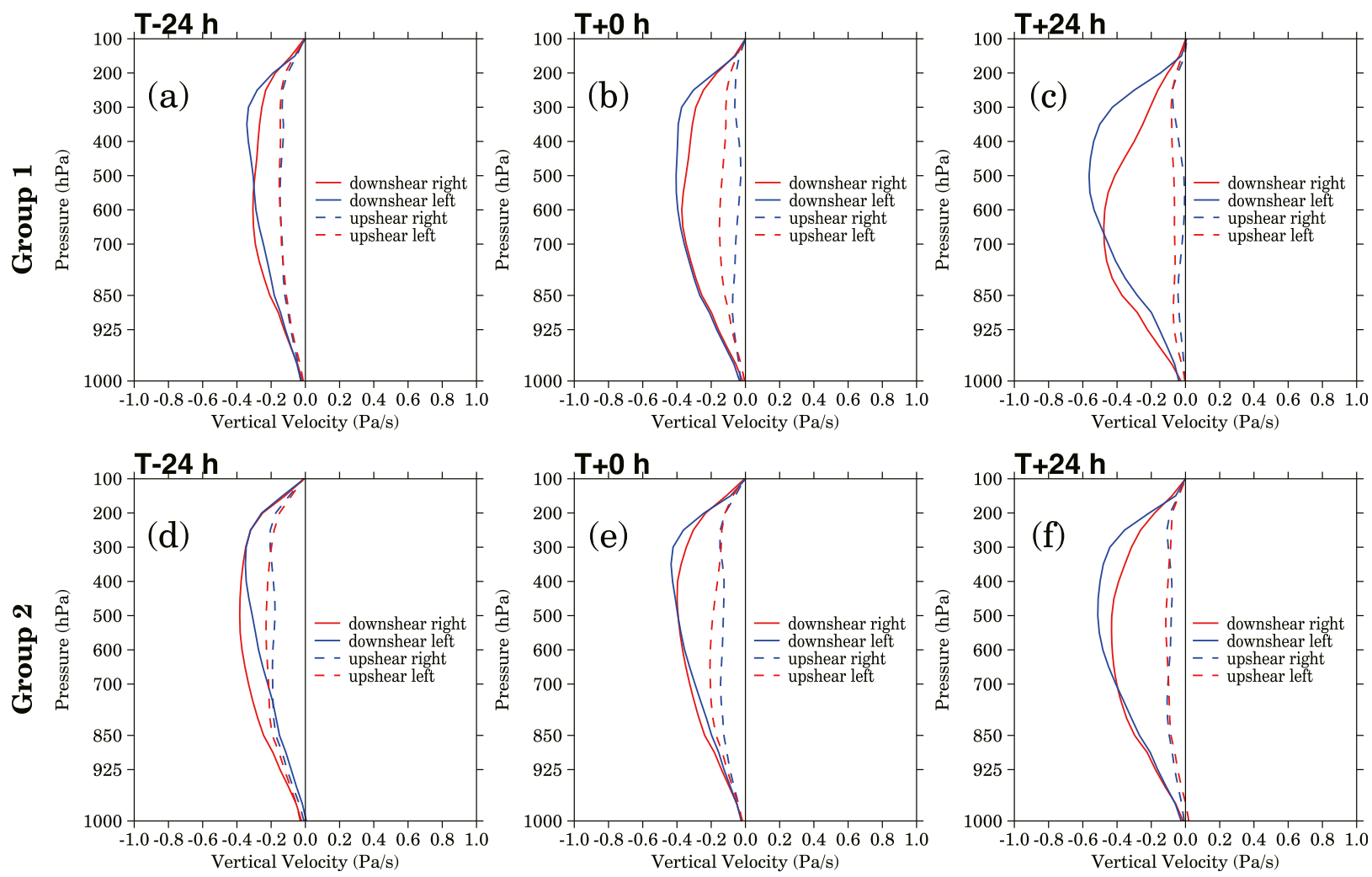

FIG. 10. Profiles of vertical motion averaged by quadrant based on the VWS direction over a 500-km radius for (top) Group 1 and (bottom) Group 2 TCs at (a),(d) $T-24 \mathrm{~h},(\mathrm{~b}),(\mathrm{e}) T+0 \mathrm{~h}$, and (c),(f) $T+24 \mathrm{~h}$

and the maintenance of upward motion and rainfall in the northeast quadrant of the TC. The dry, cold air intrusion from the northwest suppresses the convection in the northwest and southwest quadrants of the TC circulation. By comparison, the thermal advection for the Group 2 TCs is weaker, which implies that the isentropic thermodynamic uplifting is much weaker in the northeast quadrant of the TCs in Group 2 (Figs. 13d-f).

The combination of moisture advection and warm-air advection in the Group $1 \mathrm{TC}$ results in a concentrated region of enhanced potential for rainfall in the northeast quadrant to the left of track by the end of recurvature. Uplift in this region is also enhanced through isentropic uplift of this moist, warm air in the strong potential temperature gradient in the warm front region. Although the Group 2 TC has a larger envelope of high moisture, the warm front region and isentropic uplift is weaker, and the potential rainfall region associated with this mechanism is weaker and more diffuse.

\section{d. Vertical motion field and rainfall characteristics- Putting it all together}

After analyzing the major environmental conditions modulating TC convection, in this subsection we will try to put these factors together and to determine why the rainfall swaths differ for the recurving TCs in Fig. 3. For the Group $1 \mathrm{TC}$, the shear vector and motion vector become more aligned after recurvature, with the angle of separation decreasing from $142.1^{\circ}$ at $T-24 \mathrm{~h}$ to only $2.6^{\circ}$ at $T+24 \mathrm{~h}$ (Fig. 8). The upward motion in Fig. 4a is initially relatively axisymmetric prior to recurvature and is contributed to mostly by the boundary layer convergence associated with the TC motion as the shear is negligible and there is no vortex tilt at this time. The convergence is weakly concentrated in the front-right and right sections of the $\mathrm{TC}$ relative to the motion and thus there is a slight vertical motion maximum in that sector of the TC, which is also mainly the northeast quadrant (Fig. 4a). Time series of the rain rate averaged by quadrant relative to the compass heading is shown in Fig. 14. The boundary layer convergence associated with the TC motion results in a slight maximum rainfall in the northeast quadrant (Fig. 14a), which is the ROT preference for rainfall prior to actual recurvature (Fig. 5a). There is little contribution to either the vertical motion field or the moisture field inside $5^{\circ}$ of the TC center by low-level moisture or warm air advection at this time (Fig. 13a). As the TC recurves (through $T+0 \mathrm{~h}$ ), the shear vector increases in magnitude such that it begins to influence the vertical motion field resulting in a positive 

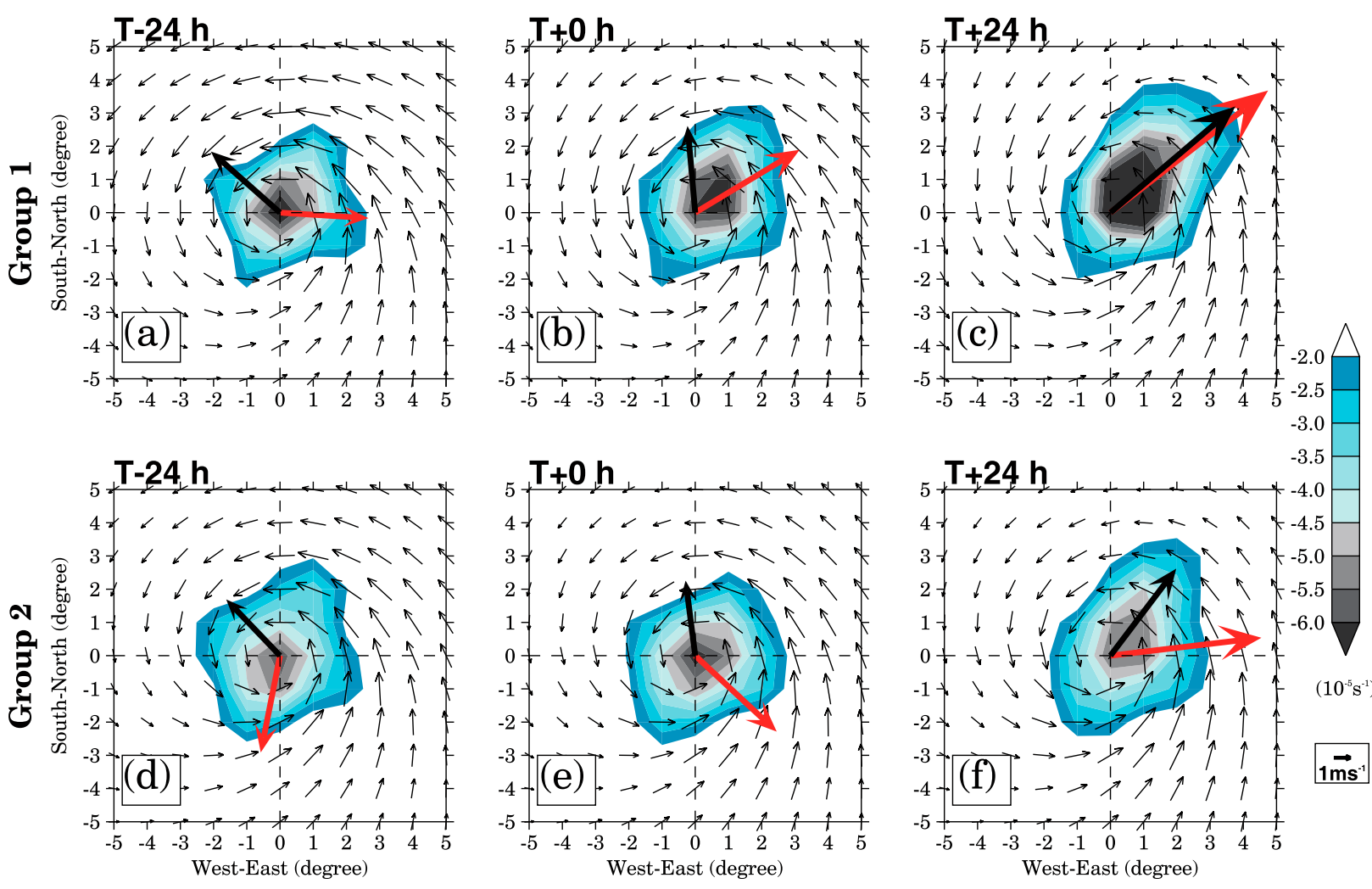

FIG. 11. As in Fig. 9, but for 950-hPa convergence (shaded; $10^{-5} \mathrm{~s}^{-1}$ ).

vertical motion asymmetry in the downshear quadrants (Fig. 4b). When combined with the front-right asymmetry induced by the TC-motion vector, this results in enhanced vertical motion and rainfall in the northeast quadrant of the TC (Fig. 14a) and a rainfall that is still ROT (Fig. 5a). Upslope of warm, moist air over the isentropes in the developing warm-frontal region also contributed to the positive vertical motion in the northeast quadrant. By the end of the recurvature period, the VWS and motion have both increased in magnitude and become aligned so that each reinforces the other positively. The maximum vertical motion is located in the downshear quadrants, which are superimposed on the front quadrants relative to motion. The VWS mechanism tends to dominate the forcing (Frank and Ritchie 2001) and so there is a downshear-left or LOT preference for the vertical motion asymmetry (Fig. 4c). The time series of quadrant-averaged rainfall relative of the TC motion vector shows that the rain switches from ROT to LOT (Fig. 5a). Finally, warm temperature and moisture advection associated with upslope over the isentropes in the warm front region within 5 degrees of the TC center have increased considerably by this time (Fig. 13c). This upslope is maximized in the same region as the VWS and motion forcing (i.e., the northeast quadrants) and also contributes to positive vertical motion, clouds, and rainfall in the left of the TC track (Fig. 14a). Overall, the comprehensive effects of these factors results in higher rainfall amount, a wider rainfall swath after recurvature, and LOT rain preference (Fig. 3a).

By comparison, the shear and motion directions are never aligned for the Group 2 TCs. The separation angle between these two vectors varies from $235^{\circ}$ at $T-24 \mathrm{~h}$ to $46.1^{\circ}$ at $T+24 \mathrm{~h}$ (Fig. 8). The ascent is initially quite axisymmetric with a slight maximum in the south quadrant due to weak VWS forcing, which results in rainfall that is slightly rotated to the south quadrant (Figs. $4 \mathrm{~d}$ and $14 \mathrm{~b}$ ), which is also LOT at $T-24 \mathrm{~h}$ (Figs. 3 and $5 \mathrm{~b}$ ). As the TC recurves (through $T+0 \mathrm{~h}$ ), the vertical motion pattern becomes meridionally elongated and maximized to the right of the track, most likely contributed to by boundary layer convergence associated with the TC motion and shear-induced asymmetry (Fig. 4e). The VWS vector moves to the right of the motion vector and the combination of these forcings results in a switch in the rainfall to the east quadrant (Fig. 14b), which is also ROT (Fig. 5b). There is no contribution to upslope due to isentropic uplift through the recurvature time as the slowly developing warm frontal region is more than $7^{\circ}$ from the TC center at this time. By $24 \mathrm{~h}$ after recurvature the motion and VWS 

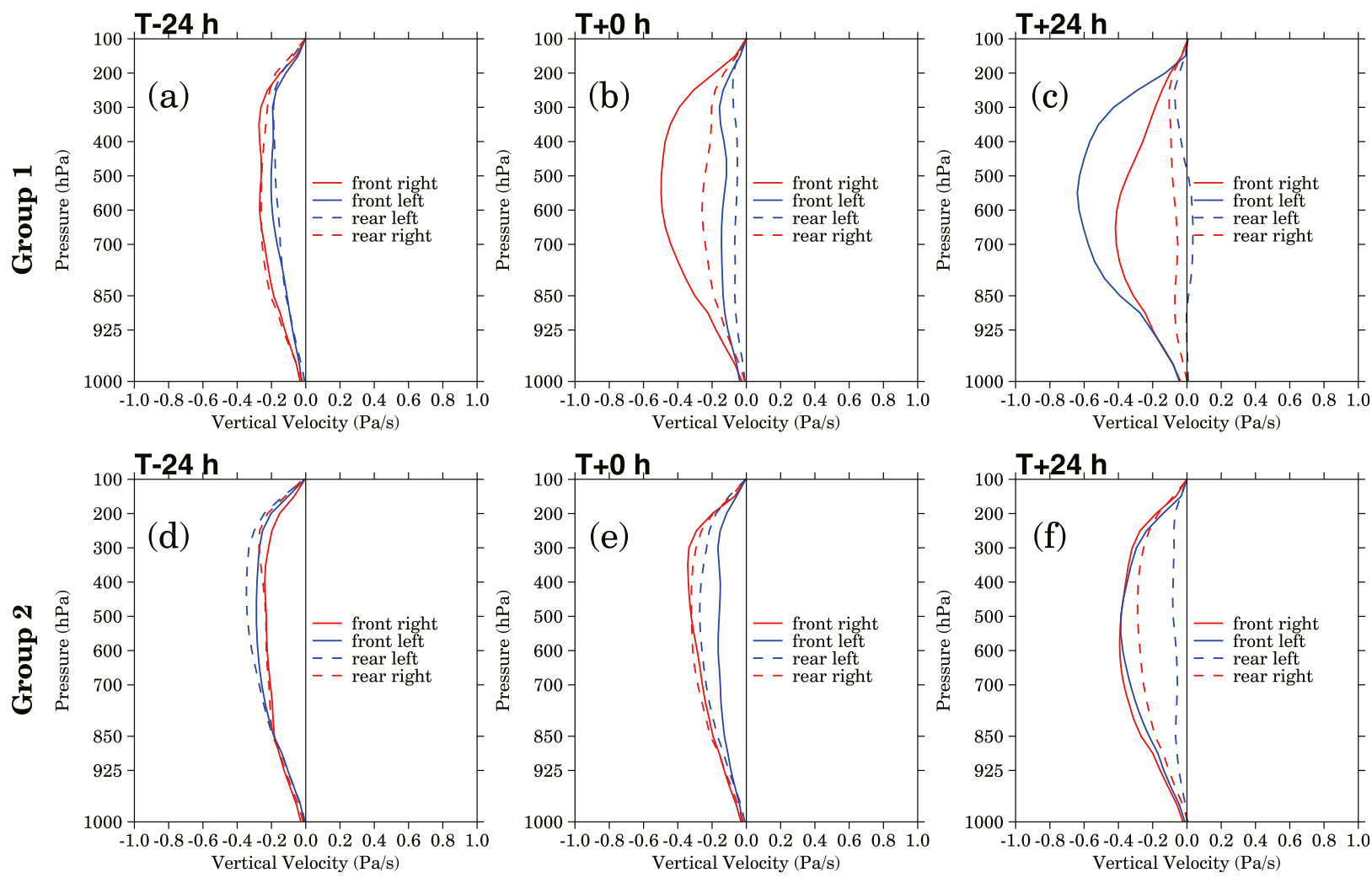

FIG. 12. As in Fig. 10, but based on the TC motion direction.

vectors are still separated by $46.1^{\circ}$, although both are pointing in an eastward direction. The shear vector contributes to vertical motion in the northeast and southeast quadrants, while the motion-related forcing contributes to vertical motion in the northeast and front quadrants. The resulting vertical motion field is spread over a larger area than for the Group 1 TC (Fig. 4f). Additional uplift over the isentropes in the weak warm front region within $5^{\circ}$ of the TC center also contributes to the vertical motion and moisture in the east quadrant of the TC. By this time, rainfall is distributed in a broad region from the northeast to the southeast (Fig. 14b) and is maximized to the ROT (Fig. 5b). These changes result in lower rainfall amounts and a smaller rainfall swath after recurvature (Fig. 3b). Moreover, the overall amount of average rainfall is higher in the Group 2 TC than the Group $1 \mathrm{TC}$ even before $T-24 \mathrm{~h}$ (black line in Fig. 14), most likely because of the larger, stronger envelope of moisture surrounding the $\mathrm{TC}$ at all times through the recurvature period.

\section{Discussion and conclusions}

The rainfall characteristics of 88 recurving TCs over the western North Pacific are investigated for the period 2004-15. The TCs are categorized into two groups based on the first EOF mode (EOF1) and first PC from a 500hPa geopotential height EOF analysis. Group 1 contains 40 TCs with $\mathrm{PC} 1>0$ and is characterized by a stronger midlatitude circulation and closer distance between the midlatitude baroclinic zone and the TC, while Group 2 contains 48 TCs with $\mathrm{PC} 1<0$ and is characterized by a weaker midlatitude baroclinic zone and more remote distance between the baroclinic zone and the TC. The two kinds of large-scale circulations result in two primary rainfall swaths during recurvature, which are a ROT-to-LOT rainfall switch for Group 1 TCs and a LOT-to-ROT switch for Group 2 TCs. The composite large-scale circulation patterns associated with each group are investigated to determine why the rainfall swaths differ.

The results show that the large-scale environmental fields are closely related to the changes in rainfall pattern for the two sets of recurving TCs. In Group 1, as the TCs recurve and become embedded in the strong baroclinic zone, a relatively strong interaction occurs between the TC and the midlatitude circulation. At the beginning of recurvature the VWS is very weak and not aligned with the TC motion vector and the pattern of upward motion is distributed in a broad region around the TC circulation, with a slight preference for rainfall in the front-right quadrant (Fig. 15a). This is in agreement 

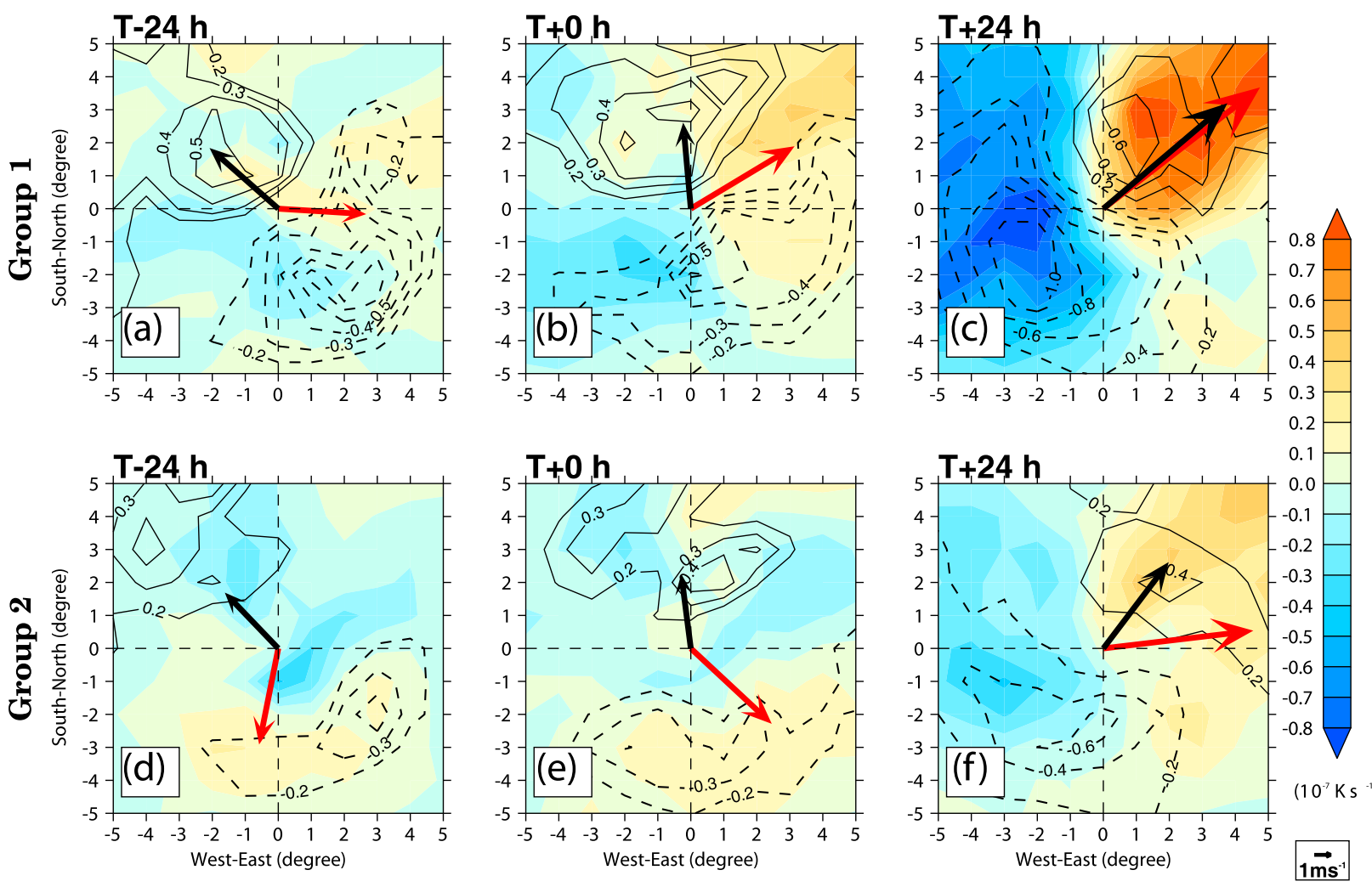

FIG. 13. As in Fig. 9, but for 700-hPa specific humidity advection (contour; $10^{-7} \mathrm{~g} \mathrm{~kg}^{1} \mathrm{~s}^{-1}$ ) and 850 -hPa thermal advection (shaded; $\left.10^{-7} \mathrm{~K} \mathrm{~s}^{-1}\right)$.

with the ideal model for rainfall in a slow-moving vortex (Shapiro 1983; Frank and Ritchie 1999). The rainfall is maximized to the ROT. By the end of recurvature (Fig. 15b), as the rapidly increasing VWS and TC motion vectors become aligned, the positive vertical motion becomes extremely focused. More rainfall is concentrated in the northeast quadrant of the TC, which is both the downshear and front quadrants, with a preference for downshear-left. The thermodynamic fields feature a strong temperature gradient. Drier, colder air advects around the west side of the TC, and moist, warmer air advects around the east of the TC

\section{Group 1}

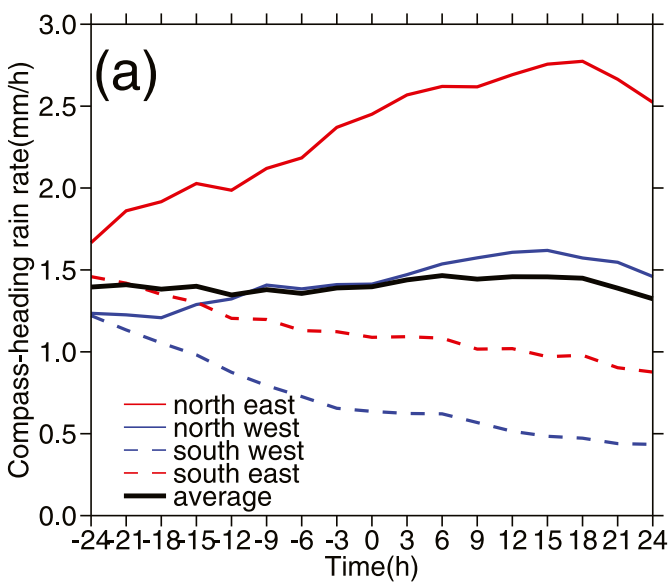

Group 2

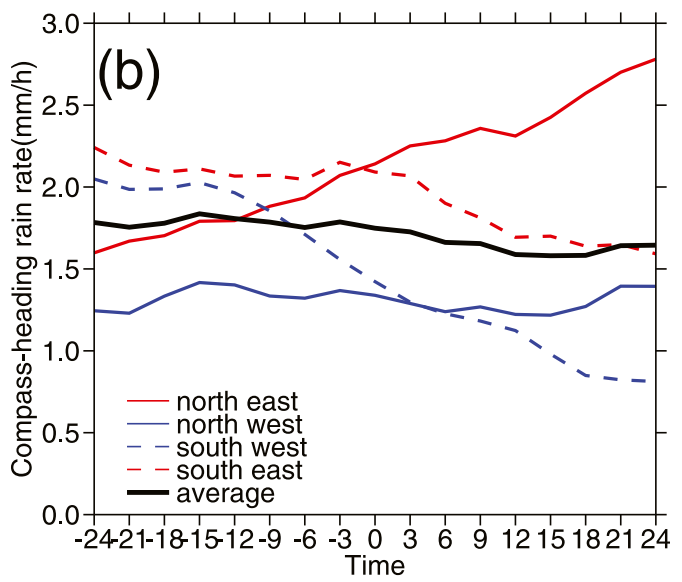

FIG. 14. The time series of rain rate averaged by quadrant within a 500-km radius for (a) Group 1 and (b) Group 2 from $T-24 \mathrm{~h}$ to $T+24 \mathrm{~h}$. 

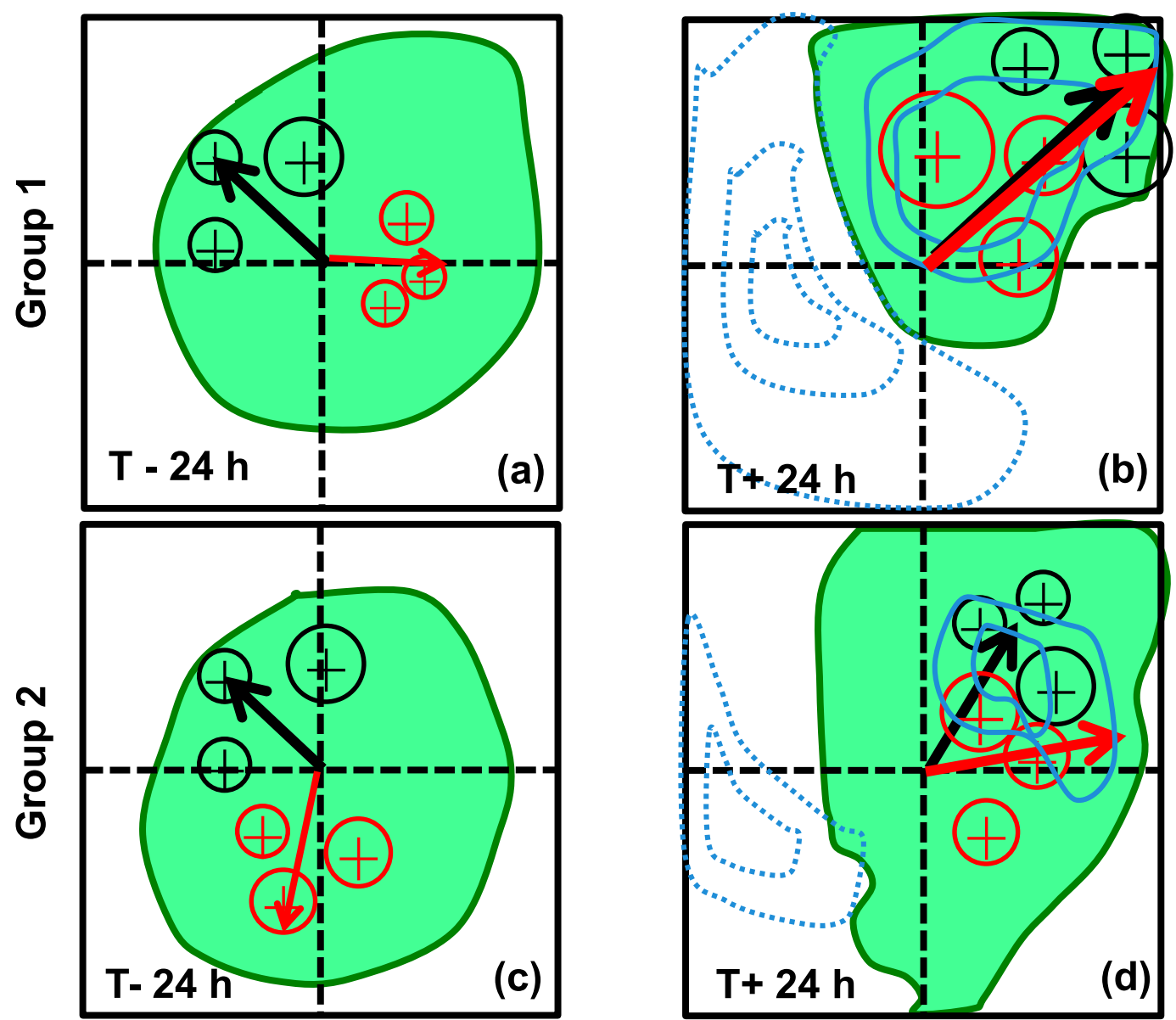

FIG. 15. Conceptual diagrams of the rainfall pattern for (top) Group 1 and (bottom) Group 2 at (a), (c) $T-24 \mathrm{~h}$ and (b),(d) $T+24 \mathrm{~h}$. The red and black arrows denote the VWS vector and motion vectors, respectively. The red plus sign denotes the shear-induced ascent. The black plus sign denotes the motion-induced ascent. The blue solid contours are regions of isentropic upslope caused by a combination of moist and warm air advection while the blue dashed contours are regions of isentropic downslope caused by the combination of dry and cold air advection. The green shading represents the rainfall areas.

circulation, similar to the patterns identified for TCs undergoing extratropical transition (e.g., Ritchie and Elsberry 2007). The strong upslope of warm, moist air along the warm front further contributes to the uplifting in the front-left quadrant of the TC. The maximum in rainfall is slightly rotated from the uplift center to the front-left quadrant as the TC vortex flows cyclonically. Therefore, the rainfall pattern changes from ROT to LOT, with rainfall concentrated in the front-left quadrant with respect to track. Overall, the comprehensive effects of these factors results in higher rainfall and a wider rainfall swath after recurvature (Fig. 3a).

By contrast, the Group 2 TC is still relatively isolated and remote from the midlatitude circulation during, and after recurving and the baroclinic zone is weaker. A relatively weaker interaction occurs between the TC and the midlatitude circulation. The magnitude of the VWS and TC motion increases less compared to the Group 1 TCs and their directions do not become as well aligned by the end of the recurvature period. The thermodynamic fields are characterized by abundant moisture from the lower latitudes, a weak baroclinic temperature gradient to the north, and a relatively weak dipole of moisture advection within the TC circulation. At the beginning of the recurvature period (Fig. 15c), the direction of the VWS vector is almost opposite to the TC motion vector $\left(235^{\circ}\right)$, and the related uplifting and rainfall pattern is quite axisymmetric. There is a slight maximum in ascent located in the southwest quadrant, which is also the downshear quadrant relative to the shear vector and rear-left quadrant relative to the motion vector. By the end of recurvature (Fig. 15d), the VWS vector and motion vector are still not aligned with the motion vector rotated approximately $46^{\circ}$ to the left of 
the VWS vector, and the vertical motion is not as concentrated as that in Group 1. Instead, the vertical motion is spread from the northeast to east to southeast quadrants. The VWS forcing contributes to the upward motion in the northeast and southeast quadrants, which are both downshear, whereas the motion vector contributes more directly to the upward motion in the northeast quadrant, which is the front quadrant with respect to TC motion. The weak warm advection along tilting of isentropic surfaces results in a relatively weaker isentropic lifting to the east of TC and likely contributes only slightly to the overall vertical motion pattern. Because the combined forcing from the VWS and motion spreads the vertical motion field across the northeast and southeast quadrants, the rainfall pattern changes from LOT to ROT during recurvature. These changes results in less rainfall and a narrower rainfall swath after recurvature compared with before recurvature (Fig. 3b). Although the dynamic and thermodynamic lifting are weaker in Group 2 than Group 1, the larger envelope of moisture in Group 2 contributes to the relatively larger overall rainfall amount compared with the rainfall in Group 1.

In contrast to many recent studies, this study focuses on how the rainfall patterns change in TCs over the western North Pacific as they approach the recurvature point and the environmental factors that impact these pattern changes. It is found that not only the VWS speed and translation speed but also the separation angle between these two vectors can significantly modulate the rainfall pattern and the preference for ROT or LOT rainfall along the TC track.

Future work includes further investigation of how the separation angle between the motion vector and the shear vector modulates the evolution of the TC rainfall. Even within the short 48-h study period used here, well before the TC transforms into an ex-TC, differences in the midlatitude circulation resulted in different separation angles and in distinct differences in the rainfall swath during recurvature. In addition, initial examination of the EOF2 and EOF3 patterns suggests that they contain information on rainfall amounts and spatial structure and thus bear further examination. Specific cases of recurving TCs, which exemplify the two groups defined here, will be examined at high resolution with a numerical model to determine how important the detailed inner core processes that accompany LOT-ROT and ROT-LOT TCs are compared with environmental influences. Following Matyas (2007) and Zick and Matyas (2016), it may also be interesting to use different spatial metrics to quantify and examine the switches of rainfall patterns.

Acknowledgments. This paper has been improved by the thoughtful comments of two anonymous reviewers.
The research was undertaken with the assistance of resources and services from the National Computational Infrastructure (NCI), which is supported by the Australian Government. The study is partly funded by the National Natural Science Foundation of China (Grants 41405057 and 41575045).

\section{REFERENCES}

Atallah, E. H., L. F. Bosart, and A. R. Aiyyer, 2007: Precipitation distribution associated with landfalling tropical cyclones over the eastern United States. Mon. Wea. Rev., 135, 2185-2206, https://doi.org/10.1175/MWR3382.1.

Bender, M. A., 1997: The effect of relative flow on the asymmetric structure in the interior of hurricanes. J. Atmos. Sci., 54, 703-724, https://doi.org/10.1175/1520-0469(1997)054<0703: TEORFO $>2.0 . \mathrm{CO} ; 2$.

Bretherton, C. S., C. Smith, and J. M. Wallace, 1992: An intercomparison of methods for finding coupled patterns in climate data. J. Climate, 5, 541-560, https://doi.org/ 10.1175/1520-0442(1992)005<0541:AIOMFF >2.0.CO;2.

Chan, J. C. L., K. S. Liu, S. E. Ching, and E. S. T. Lai, 2004: Asymmetric distribution of convection associated with tropical cyclones making landfall along the south China coast. Mon. Wea. Rev., 132, 2410-2420, https://doi.org/ 10.1175/1520-0493(2004)132<2410:ADOCAW>2.0.CO;2.

Chen, S. S., J. A. Knaff, and F. D. Marks, 2006: Effects of vertical wind shear and storm motion on tropical cyclone rainfall asymmetries deduced from TRMM. Mon. Wea. Rev., 134, 3190-3208, https://doi.org/10.1175/MWR3245.1.

Corbosiero, K. L., and J. Molinari, 2002: The effects of vertical wind shear on the distribution of convection in tropical cyclones. Mon. Wea. Rev., 130, 2110-2123, https://doi.org/ 10.1175/1520-0493(2002)130<2110:TEOVWS >2.0.CO;2.

, and - 2003: The relationship between storm motion, vertical wind shear, and convective asymmetries in tropical cyclones. J. Atmos. Sci., 60, 366-376, https://doi.org/10.1175/ 1520-0469(2003)060<0366:TRBSMV >2.0.CO;2.

Frank, W. M., and E. A. Ritchie, 1999: Effects of environmental flow upon tropical cyclone structure. Mon. Wea. Rev., 127, 2044-2061, https://doi.org/10.1175/1520-0493(1999)127<2044: EOEFUT $>2.0 . \mathrm{CO} ; 2$.

, and - 2001: Effects of vertical wind shear on the intensity and structure of numerically simulated hurricanes. Mon. Wea. Rev., 129, 2249-2269, https://doi.org/10.1175/ 1520-0493(2001)129<2249:EOVWSO>2.0.CO;2.

Halverson, J., G. M. Heymsfield, J. Simpson, H. Pierce, T. Hock, and E. A. Ritchie, 2006: Warm core structure of Hurricane Erin diagnosed from high-altitude dropsondes during CAMEX-4. J. Atmos. Sci., 63, 309-324, https://doi.org/10.1175/JAS3596.1.

Harr, P. A., R. L. Elsberry, and T. F. Hogan, 2000: Extratropical transition of tropical cyclones over the western North Pacific. Part II: The impact of midlatitude circulation characteristics. Mon. Wea. Rev., 128, 2634-2653, https://doi.org/ 10.1175/1520-0493(2000)128<2634:ETOTCO > 2.0.CO;2.

Heymsfield, G. M., J. Halverson, E. A. Ritchie, J. Simpson, J. Molinari, and L. Tian, 2006: Structure of the highly sheared Tropical Storm Chantal during CAMEX-4. J. Atmos. Sci., 63, 268-287, https://doi.org/10.1175/JAS3602.1.

Huffman, G. J., R. F. Adler, M. Morrissey, D. T. Bolvin, S. Curtis, R. Joyce, B. McGavock, and J. Susskind, 2001: Global precipitation at one-degree daily resolution from multisatellite 
observations. J. Hydrometeor., 2, 36-50, https://doi.org/ 10.1175/1525-7541(2001)002<0036:GPAODD > 2.0.CO;2.

— , and Coauthors, 2007: The TRMM Multisatellite Precipitation Analysis (TMPA): Quasi-global, multiyear, combined-sensor precipitation estimates at fine scales. J. Hydrometeor., 8, 38-55, https://doi.org/10.1175/JHM560.1.

Jiang, H., J. B. Halverson, and J. Simpson, 2008a: On the differences in storm rainfall from Hurricanes Isidore and Lili. Part I: Satellite observations and rain potential. Wea. Forecasting, 23, 29-43, https://doi.org/10.1175/2007WAF2005096.1.

, — — - and E. J. Zipser, 2008b: On the differences in storm rainfall from Hurricanes Isidore and Lili. Part II: Water budget. Wea. Forecasting, 23, 44-61, https://doi.org/10.1175/ 2007WAF2005120.1.

Jones, S., 1995: The evolution of vortices in vertical shear. I: Initially barotropic vortices. Quart. J. Roy. Meteor. Soc., 121 821-851, https://doi.org/10.1002/qj.49712152406.

- 2000: The evolution of vortices in vertical shear. III: Baroclinic vortices. Quart. J. Roy. Meteor. Soc., 126, 3161-3185, https://doi.org/10.1002/qj.49712657009.

_ and Coauthors, 2003: The extratropical transition of tropical cyclones: Forecast challenges, current understanding and future directions. Wea. Forecasting, 18, 1052-1092, https://doi.org/ 10.1175/1520-0434(2003)018<1052:TETOTC >2.0.CO;2.

Kitabatake, N., 2008: Extratropical transition of tropical cyclones in the western North Pacific: Their frontal evolution. Mon. Wea. Rev., 136, 2066-2090, https://doi.org/10.1175/2007MWR1958.1.

Klein, P. M., P. A. Harr, and R. L. Elsberry, 2000: Extratropical transition of western North Pacific tropical cyclones: An overview and conceptual model of the transformation stage. Wea. Forecasting, 15, 373-395, https://doi.org/10.1175/ 1520-0434(2000)015<0373:ETOWNP > 2.0.CO;2.

Kofron, D. E., E. A. Ritchie, and J. S. Tyo, 2010a: Determination of a consistent time for the extratropical transition of tropical cyclones. Part I: Examination of existing methods for finding "ET time." Mon. Wea. Rev., 138, 4328-4343, https://doi.org/10.1175/ 2010MWR3180.1.

,-- , and $-2010 \mathrm{~b}$ : Determination of a consistent time for the extratropical transition of tropical cyclones. Part II: Potential vorticity metrics. Mon. Wea. Rev., 138, 4344-4361, https://doi.org/10.1175/2010MWR3181.1.

Kummerow, C., and Coauthors, 2001: The evolution of the Goddard Profiling Algorithm (GPROF) for rainfall estimation from passive microwave sensors. J. Appl. Meteor., 40, 1801-1820, https://doi.org/10.1175/1520-0450(2001)040<1801: TEOTGP $>2.0 . \mathrm{CO} ; 2$.

Lonfat, M., F. M. Marks, and S. Y. Chen, 2004: Precipitation distribution in tropical cyclones using the Tropical Rainfall Measuring Mission (TRMM) microwave imager: A global perspective. Mon. Wea. Rev., 132, 1645-1660, https://doi.org/ 10.1175/1520-0493(2004)132<1645:PDITCU>2.0.CO;2.

Madala, R. V., and S. A. Piacsek, 1975: Numerical simulation of asymmetric hurricanes on a $\beta$-plane with vertical shear. Tellus, 27, 453-468, https://doi.org/10.3402/tellusa.v27i5.10172.

Matyas, C., 2007: Quantifying the shapes of U.S. landfalling tropical cyclone rain shields. Prof. Geogr., 59, 158-172, https://doi.org/ 10.1111/j.1467-9272.2007.00604.x.
NCEP, 2000: NCEP FNL Operational Model Global Tropospheric Analyses, continuing from July 1999 (updated daily). Research Archive at the National Center for Atmospheric Research, Computational and Information Systems Laboratory, accessed 15 July 2016, https://doi.org/10.5065/ D6M043C6.

North, G. R., T. L. Bell, R. F. Cahalan, and F. J. Moeng, 1982: Sampling errors in the estimation of empirical orthogonal functions. Mon. Wea. Rev., 110, 699-706, https://doi.org/ 10.1175/1520-0493(1982)110<0699:SEITEO > 2.0.CO;2.

Peng, S. M., B. F. Jeng, and R. T. Williams, 1999: A numerical study on tropical cyclone intensification. Part I: Beta effect and mean flow effect. J. Atmos. Sci., 56, 1404-1423, https://doi.org/ 10.1175/1520-0469(1999)056<1404:ANSOTC $>2.0 . C O ; 2$.

Ritchie, E. A., and R. L. Elsberry, 2001: Simulations of the transformation stage of the extratropical transition of tropical cyclones. Mon. Wea. Rev., 129, 1462-1480, https://doi.org/ 10.1175/1520-0493(2001)129<1462:SOTTSO > 2.0.CO;2.

— , and - 2007: Simulations of the extratropical transition of tropical cyclones: Phasing between the upper-level trough and tropical cyclones. Mon. Wea. Rev., 135, 862-876, https://doi.org/ 10.1175/MWR3303.1.

_ tropical cyclones and an environment with a variable Coriolis parameter. Mon. Wea. Rev., 135, 1889-1905, https://doi.org/ 10.1175/MWR3359.1.

Rogers, R., S. Chen, J. Tenerelli, and H. Willoughby, 2003: A numerical study of the impact of vertical shear on the distribution of rainfall in Hurricane Bonnie (1993). Mon. Wea. Rev., 131, 1577-1599, https://doi.org/10.1175//2546.1.

Shaffer, A. R., and R. L. Elsberry, 1982: A statistical-climatological tropical cyclone track prediction technique using as EOF representation of the synoptic forcing. Mon. Wea. Rev., 110, 1945-1955, https://doi.org/10.1175/1520-0493(1982)110<1945: ASCTCT>2.0.CO;2.

Shapiro, L. J., 1983: The asymmetric boundary layer flow under a translating hurricane. J. Atmos. Sci., 40, 1984-1998, https://doi.org/ 10.1175/1520-0469(1983)040<1984:TABLFU>2.0.CO;2.

Sinclair, M., 2004: Extratropical transition of southwest tropical cyclones. Part II: Midlatitude circulation characteristics. Mon. Wea. Rev., 132, 2145-2168, https://doi.org/10.1175/ 1520-0493(2004)132<2145:ETOSPT > 2.0.CO;2.

Stovern, D. R., and E. A. Ritchie, 2016: Analysis of environments associated with TC size and structure changes in the North Atlantic basin. 32nd Conf. on Hurricanes and Tropical Meteorology, San Juan, Puerto Rico, Amer. Meteor. Soc., 15A.7, https:// ams.confex.com/ams/32Hurr/webprogram/Paper293607.html.

Wood, K. M., and E. A. Ritchie, 2014: A 40-year climatology of extratropical transition in the eastern North Pacific. J. Climate, 27, 5999-6015, https://doi.org/10.1175/JCLI-D-13-00645.1.

— , and 2015: A definition for rapid weakening in the North Atlantic and eastern North Pacific. Geophys. Res. Lett. 42, 10 091-10 097, https://doi.org/10.1002/2015GL066697.

Zick, S. E., and C. J. Matyas, 2016: A shape metric methodology for studying the evolving geometries of synoptic-scale precipitation patterns in tropical cyclones. Ann. Assoc. Amer. Geogr., 106, 1217-1235, https://doi.org/10.1080/24694452.2016.1206460. 